\title{
Information acquisition, referral, and organization
}

\author{
Simona Grassi* \\ and \\ Ching-to Albert Ma**
}

Each of two experts may provide a service to a client. Experts' cost comparative advantage depends on an unknown state, but an expert may exert effort to get a private signal about it. In a market, an expert may refer the client to the other for a fee. In equilibrium, only one expert exerts effort and refers, and the equilibrium allocation is inefficient. Referral efficiency can be restored when experts form an organization, in which a referring expert must bear the referred expert's cost. However, the referred expert shirks from work effort because of the lack of cost responsibility.

\section{Introduction}

We study an economic system consisting of experts who provide services to clients. An expert may invest in effort to find out a client's state-contingent service costs, as well as to reduce overall costs. We consider efficiencies in a referral market and within an expert organization that can assign cost responsibilities. For each institution, we study an expert's information-acquisition and cost-reduction incentives, and experts' incentives to refer clients to each other.

Information acquisition and task assignment are topical in policy forums. In the US healthcare reform, cost-control measures are being phased in after the Affordable Care Act took effect in 2014. The Center for Medicare and Medicaid Services, the federal agency that administers the insurance programs for the elderly and the indigent, has been encouraging providers (such as general practitioners, specialists, and hospitals) to form so-called Accountable Care Organizations (ACOs). ${ }^{1}$ Such organizations are supposed to reduce cost through better care coordination

\footnotetext{
*Université de Lausanne; simona.grassi@unil.ch.

** Boston University; ma@bu.edu.

We thank the Editor, David Martimort, and two referees for giving us very valuable suggestions. For their comments, we thank Francesca Barigozzi, Jacopo Bizzotto, Jean-Philippe Bonardi, Giacomo Calzolari, Mark Dusheiko, George Georgiadis, Katharina Huesmann, Izabella Jelovac, Andrew Jones, Liisa Laine, Henry Mak, Debby Minehart, Marco Ottaviani, Raphael Parchet, Peter Zweifel, and many participants at seminars and conferences where we presented the article. Grassi is indebted to the Commonwealth Fund, the Careum Foundation, and to her mentor, Joe Newhouse, while being a Harkness/Careum Fellow at the Harvard Medical School.

${ }^{1}$ For a description of ACOs, see www.cms.gov/medicare/medicare-fee-for-service-payment/sharedsavings program/downloads/aco-narrativemeasures-specs.pdf.
} 
achieved by referrals among physicians (see Song, Sequist, and Barnett, 2014). Other professionals, such as accountants and lawyers, refer clients to each other, whether they operate in the market or within an organization. How do experts' performances compare in the market and within an organization? We provide a framework for these comparisons.

In our model, each of a set of clients would like to obtain service from one of two experts. A client's case can be easy or complicated. An easy case is always less expensive to service than a complicated one. However, the two experts have different cost comparative advantages: Expert 1 has a lower service cost than Expert 2 if the case is easy; conversely, Expert 2 incurs a lower cost than Expert 1 if the case is complicated. The complexity of a client's case is unknown. An expert may exert some effort to obtain information about the case. The effort generates an informative signal, and, as a convention, a higher signal indicates a higher likelihood of a complicated case, so a higher (expected) cost. The service from an expert gives a fixed benefit to a client, and each client pays a fixed tariff for the service. ${ }^{2}$

We first study how experts operate in a referral market. After, say, Expert 1 has exerted an effort and observed a signal, he may make a referral offer to Expert 2: the client and the service tariff are transferred from Expert 1 to Expert 2 if Expert 2 pays a referral price. The problems facing these experts are (i) effort is hidden action, unknown to anyone except the expert who exerts it, and (ii) the signal generated by effort is hidden information, unknown to anyone except the expert who has exerted the effort.

Despite asymmetric-information problems, there is an equilibrium in which Expert 1 exerts effort, and successfully refers clients to Expert 2 if and only if their signals are above a threshold (a higher signal indicating a higher expected cost). An expert's incentive is to avoid complicated and costly clients, so in equilibrium, Expert 2 only gets lemons from Expert 1 . However, Expert 2 has a cost advantage in complicated cases. Expert 1 credibly exploits this cost advantage when setting the referral price, so the referred lemons will be accepted.

Expert 2's acceptance decision is based on comparing the referral price with the average cost given that signals are above the threshold. For efficiency, Expert 2 should have compared the referral price with the actual expected cost, but this is Expert 1's private information. This discrepancy is common in adverse-selection models. As a result, Expert 1's referral and information-acquisition decisions do not internalize all cost savings due to cost comparative advantage, and are never first best.

Expert 2's equilibrium strategy, however, is completely different. He will neither exert effort nor make any referral. The cost comparative advantage for Expert 1 is for the client with an easy case, but there is no equilibrium in which Expert 2 refers a client to Expert 1. A simple case is more profitable than a complicated case. If in equilibrium Expert 2 was successful at referring a client at a signal, he would also refer the client if the signal had become higher (indicating a higher cost). In other words, Expert 2 would always refer lemons, never peaches. Expert 2's referral, therefore, would not let Expert 1 exploit his cost comparative advantage. Without any success in referral in equilibrium, Expert 2 does not exert effort.

We then study expert organizations. The referral market equilibrium is inefficient because an expert is unconcerned about the cost consequence which will be borne by the expert who accepts the referral. Our premise is that an organization differs from the market because it can make cost information available ex post. In an organization, when an expert refers a client, the referring expert can be held responsible for the cost incurred by the referred expert. We call this the cost-transfer protocol. An expert now can fully internalize benefits of cost comparative advantage. If Expert 1's signal indicates that Expert 2 has a lower expected cost, he simply refers the client to Expert 2 and, under the cost-transfer protocol, reaps the cost savings (Song, Sequist, and Barnett, 2014, identify an ACO exactly as an organization in which "physicians ... share the consequences of each other's referral decisions").

${ }^{2}$ A stylized example is this. A consumer needs to file a tax return. Simple returns are less time-consuming than complex returns. However, a tax preparer is more cost effective than an accountant for a simple return, and vice versa. 
We also examine a drawback of the cost-transfer protocol. We enrich our model by allowing each expert to choose a cost-reduction effort when serving a client. This adds another hidden action. When experts operate in the market, each is responsible for his cost, so cost effort must be efficient. Cost reduction is orthogonal to information acquisition and referral in the market. This is no longer true for an organization that uses the cost-transfer protocol.

Our point is that the cost-transfer protocol introduces a new trade-off. When experts can reduce their costs, the magnitude of cost saving determines whether a market performs better than an organization. If cost saving by effort is small, cost comparative advantage dominates cost effort, so an expert organization performs better than the market. If cost saving by effort is large, the opposite is true. Ours is also a theory about whether referrals should be among experts within a firm under the cost-transfer protocol, or among independent experts in the market.

We consider various extensions of the basic model. First, we discuss constraints on experts' capacities, and variable returns. We qualify how various results should be properly interpreted. Second, we endogenize clients' tariffs by a Bertrand game. Finally, we let cost comparative advantage potentially be big so that a client may be a lemon to one expert, but a peach to another. There, we show that referrals by both experts may arise in equilibrium.

Our article is related to the literatures on credence goods, referrals, and organizations. In contrast to models of credence goods (see the Dulleck and Kerschbamer, 2006 survey), we simplify experts' price and treatment decisions. Here, an expert sets one price and may have control over cost distributions. Furthermore, many models of credence goods are on interactions between experts and clients. Instead, we study the interactions between experts via referral and information acquisition.

Garicano and Santos (2004) study referrals between two experts who have different productivities and costs in generating revenue from a project by exerting efforts. An expert can choose between implementing a project himself, or referring it to the other expert. Referral of a project is subject to asymmetric information because a project's potential can be either high or low, which is privately known by an expert. Equilibrium referrals via fixed price or revenue-sharing contracts are often inefficient. In our model, private information is in the form of a continuous signal, rather than a binary signal. The kinds of inefficiency in our model are also different. First, experts' efforts to acquire information are inefficient. Second, an expert's equilibrium referrals do not internalize social cost savings. Third, when experts form an organization, we allow the transfer of costs, which leads to shirking.

Referrals incentives have been studied in models of consumers searching for experts' advice; see Arbatskaya and Konishi (2012), Bolton, Freixas, and Shapiro (2007), Inderst and Ottaviani (2009), and Park (2005). Experts face a trade-off between honestly advising clients to build a good reputation, and reaping a quick profit at the client's expense. We do not model search or reputation here but show that even without threats from consumers, referrals may occur.

Referrals are studied in the health literature. In the health sector, insurers set up incentive mechanisms for referrals between providers, say, between general practitioners and specialists (see Mariñoso and Jelovac, 2003; Shumsky and Pinker, 2003). We do not follow a contract-design approach, but our result suggests that physician organizations may lead to efficient referrals. Also, market referrals with financial transfers are uncommon in the medical sector. However, our analysis of how an organization provides incentive to refer clients is relevant to the organizational approach currently advocated in the health domain. We will revisit this after we have presented results.

We contribute to organizational economics. Our hypothesis that costs become transferable when experts merge is similar to the reallocation of ownership rights within a firm. Schmidt (1996) argues that the allocation of ownership rights has an important impact on the allocation of information about the firm. Garicano (2000), Garicano and Santos (2004), and Fuchs and Garicano (2010) argue that organizations can better match clients to experts, and this is supported by evidence of obstetric practices in Epstein, Ketcham, and Nicholson (2010). We have argued 
that when cost comparative advantage is internalized, matches will be efficient, so we explain why better matches happen.

However, we point out the degradation of work incentives when costs are transferred in an organization. This possibility has also been raised by Frandsen and Rebitzer (2015), who show that free-riding problems in ACOs may erode savings from better care coordination. Cebul et al. (2008) and Rebitzer and Votruba (2011) provide evidence on the adverse effects of coordination failures in the healthcare delivery system in the United States. The free-riding and work-incentive deficiency should be weighed against better referrals, which, according to Able (2013), is the mechanism by which ACOs reduce aggregate medical expenditures and improve Medicare patients' health.

The article is organized as follows. In Section 2, we set up the model and derive the first best. Section 3 studies a market in which experts can refer clients to each other at a price. In Section 4, we present organizations and compare them to the market and the first best. We also provide specific perspectives on the relevance of our theory to legal and medical professionals. In Section 5, we consider a number of robustness issues. Section 6 concludes. An Appendix contains proofs of results.

\section{The model}

Clients and experts. Each of a set of clients needs the service from one of two experts. These clients may be consumers who seek services from professionals such as lawyers, doctors, or engineers. Alternatively, a company may have projects that require inputs from outside contractors, and these projects correspond to the clients, whereas the contractors are the experts. We let there be a continuum of clients, with the total mass normalized at 1 . Each client is characterized by a state or a type. Each client's state or type is independently and identically distributed on the binary support $\left\{\omega_{1}, \omega_{2}\right\}$ with a probability $1 / 2$ on each state. We discuss the equal prior assumption in Section 5.

There are two experts, namely, Expert 1 and Expert 2. Each expert can provide a service to any number of clients. This amounts to an assumption that experts have enough capacities. We further assume that the cost of service (including effort disutility, see below) is linear in the number of clients served. We do not aim to construct a theory on organizations and incentives based on returns to scale or fixed costs, so nonbinding capacity and constant returns are natural assumptions. We assume that each expert gives the same benefit to a client.

Experts differ by their service costs that are dependent on a client's states. The following table defines each expert's cost contingent on a client's type:

\begin{tabular}{lcc}
\hline & State $\omega_{1}$ & State $\omega_{2}$ \\
\hline Expert 1's cost & $c_{L}$ & $c_{H}$ \\
Expert 2's cost & $c_{L}+\Delta$ & $c_{H}-\Delta$ \\
\hline
\end{tabular}

where $0<c_{L}<c_{L}+\Delta<c_{H}-\Delta<c_{H}$ (so $2 \Delta<c_{H}-c_{L}$ ). If a client's state is $\omega_{1}$, Expert 1's service cost, $c_{L}$, is lower than Expert 2's, $c_{L}+\Delta$, but if a client's state is $\omega_{2}$, Expert 2's service cost is lower. (In Section 5, we consider an alternative cost configuration: $0<c_{L}<c_{H}-\Delta<c_{L}+\Delta<c_{H}$.)

The cost saving $\Delta$ is assumed to be symmetric between the experts for convenience. Ex ante, each expert has the same expected cost of providing services to clients. State $\omega_{1}$ can be thought of as a "good" or "easy" state: the service cost is lower, either $c_{L}$ for Expert 1 or $c_{L}+\Delta$ for Expert 2. State $\omega_{2}$ corresponds to a "bad" or "complicated" state with service cost either $c_{H}-\Delta$ or $c_{H}$. Expert 1 has a cost advantage $\Delta$ in state $\omega_{1}$, whereas Expert 2 has an advantage in state $\omega_{2}$. Finally, when an expert does not service a client, he has an outside option which is normalized at 0 .

The setup for experts' costs will be enriched in Section 4. Each expert's cost will be stochastic, and each expert can take an effort to reduce the expected value of his cost distribution (so the costs 
defined above would be expected values). We will then use the more general setup to compare markets and organizations. Until then, we use the simpler setup above. Our definition of the first best, and our results in Section 3 are unaffected by the omission of cost-reduction efforts.

We subscribe to the credence-good framework. Clients do not get to observe their states when they seek services from experts. Neither do clients get to observe how much cost an expert eventually incurs to provide the service. The only contractible event for clients is that the service is provided. To a client, for a given tariff for service provided, the experts are identical because each of them provides the same benefit.

Information acquisition. Experts do not observe clients' cost types. Each expert can acquire information about a client's cost type by exerting effort. We assume that each expert has the same information-acquisition technology and effort disutility. The information comes in the form of a signal defined on a support, $s \in[\underline{s}, \bar{s}]$. Let $e \in \mathbb{R}_{+}$denote an expert's effort, and $\phi(e)$ denote the disutility of effort. The disutility function $\phi$ is increasing and convex, and satisfies the usual Inada conditions. ${ }^{3}$

Let $f_{i}(s \mid e)$ be the density of the signal $s$ conditional on effort $e$ and state $\omega_{i}, i=1,2$. We assume that both $f_{1}$ and $f_{2}$ are always strictly positive, and continuous. By Bayes' rule, conditional on a signal $s$, the posterior probability of the state being $\omega_{i}$ is

$$
\operatorname{Pr}\left(\omega_{i} \mid s, e\right)=\frac{f_{i}(s \mid e)}{f_{1}(s \mid e)+f_{2}(s \mid e)}, \quad i=1,2 .
$$

We assume that for any effort, the signal satisfies Monotone Likelihood Ratio Property (MLRP):

$$
\frac{f_{2}\left(s^{\prime} \mid e\right)}{f_{2}(s \mid e)} \geq \frac{f_{1}\left(s^{\prime} \mid e\right)}{f_{1}(s \mid e)} \text { for } s^{\prime}>s, \text { each } e .
$$

As a normalization, we let the signals be completely uninformative at the lowest effort, $e=0$, so that $f_{1}(s \mid 0)=f_{2}(s \mid 0)$, each $s \in[s, \bar{s}]$, and that for $e>0$, the inequality in the MLRP definition holds as a strict inequality for each $s$. Under MLRP, $\frac{f_{2}(s \mid e)}{f_{1}(s \mid e)}$ is increasing in $s$, so a higher value of the signal indicates a higher likelihood that the state is $\omega_{2}$ :

$$
\operatorname{Pr}\left(\omega_{2} \mid s, e\right)=\frac{1}{1+\frac{f_{1}(s \mid e)}{f_{2}(s \mid e)}} \quad \text { is increasing in } s .
$$

For future use, we note that the ex ante density of signal $s$, given effort $e$, is $\operatorname{Pr}\left(\omega_{1}\right) f_{1}(s \mid e)+$ $\operatorname{Pr}\left(\omega_{2}\right) f_{2}(s \mid e)=0.5\left[f_{1}(s \mid e)+f_{2}(s \mid e)\right]$.

A higher effort makes signals more informative. We use the following assumption on how the densities $f_{1}$ and $f_{2}$ relate to efforts, and call it the Informativeness Property:

For $e^{\prime}>e, f_{2}\left(s \mid e^{\prime}\right)$ first-order stochastically dominates $f_{2}(s \mid e)$, and $f_{1}(s \mid e)$ first-order stochastically dominates $f_{1}\left(s \mid e^{\prime}\right)$.

A higher effort reduces the conditional cumulative density $\int_{\underline{s}}^{s} f_{2}(x \mid e) \mathrm{d} x$ and raises the conditional cumulative density $\int_{s}^{s} f_{1}(x \mid e) \mathrm{d} x$. First-order stochastic dominance is often used in the literature to define how effort affects information. A higher effort makes a lower signal more indicative of state $\omega_{1}$, whereas it makes a higher signal more indicative of state $\omega_{2}$. We further assume that both conditional densities are differentiable in $e$.

First best. An allocation is an effort to be taken by an expert, and a decision rule that assigns a client to an expert according to the generated signal. The first best is an allocation that

${ }^{3}$ We rule out multiple information-acquisition efforts by an expert. This may be due to high fixed cost for each round of information acquisition. 
minimizes experts' expected service cost and effort disutilities. In Section 4, we will extend the definition of an allocation and the first best to include a cost-reduction effort.

Let an expert take effort $e$. Contingent on signal $s$, the expected cost of servicing this client by Experts 1 and 2 are, respectively,

$$
\begin{gathered}
\operatorname{Pr}\left(\omega_{1} \mid s, e\right) c_{L}+\operatorname{Pr}\left(\omega_{2} \mid s, e\right) c_{H} \\
\operatorname{Pr}\left(\omega_{1} \mid s, e\right)\left(c_{L}+\Delta\right)+\operatorname{Pr}\left(\omega_{2} \mid s, e\right)\left(c_{H}-\Delta\right) .
\end{gathered}
$$

The conditional probabilities are given by (1), so Expert 2 has a cost lower than Expert 1 if and only if $f_{1}(s \mid e) \leq f_{2}(s \mid e)$. For each effort $e$, define $\widehat{s}^{f b}(e)$ by $f_{1}\left(\widehat{s}^{f b} \mid e\right)=f_{2}\left(\widehat{s}^{f b} \mid e\right)$. By MLRP, $s \geq \widehat{s}^{f b}$ if and only if $f_{1}(s \mid e) \leq f_{2}(s \mid e)$. In this notation, the cost-minimizing allocation assigns a client to Expert 2 if and only if the client's signal $s$ is larger than $\widehat{s}^{f b}(e)$.

Given the cost-minimizing allocation, the total expected service cost and effort disutility per client is

$$
\begin{gathered}
0.5 \int_{\underline{s}}^{\widehat{s}^{f b}(e)}\left\{\operatorname{Pr}\left(\omega_{1} \mid x, e\right) c_{L}+\operatorname{Pr}\left(\omega_{2} \mid x, e\right) c_{H}\right\}\left[f_{1}(x \mid e)+f_{2}(x \mid e)\right] \mathrm{d} x+ \\
0.5 \int_{\widehat{s}^{f b}(e)}^{\bar{s}}\left\{\operatorname{Pr}\left(\omega_{1} \mid x, e\right)\left(c_{L}+\Delta\right)+\operatorname{Pr}\left(\omega_{2} \mid x, e\right)\left(c_{H}-\Delta\right)\right\}\left[f_{1}(x \mid e)+f_{2}(x \mid e)\right] \mathrm{d} x+\phi(e) .
\end{gathered}
$$

We assume that (4) is quasiconvex. ${ }^{4}$ The first-best effort, $e^{f b}$, is one that minimizes (2). The first-order condition is:

$$
0.5 \Delta \int_{\bar{s}^{f b}(e f b)}^{\bar{s}}\left\{\frac{\partial f_{2}\left(x \mid e^{f b}\right)}{\partial e}-\frac{\partial f_{1}\left(x \mid e^{f b}\right)}{\partial e}\right\} \mathrm{d} x=\phi^{\prime}\left(e^{f b}\right) .
$$

The first-best characterization has the following interpretations. First, the base costs, $c_{L}$ and $c_{H}$, set up reference points only, so their values do not appear in the first-order condition (5). Second, cost saving, from $c_{H}$ to $c_{H}-\Delta$ may be achieved, and cost increase from $c_{L}$ to $c_{L}+\Delta$ may be avoided. The assignment of a client to Expert 2 whenever $s$ is above a threshold is for cost effectiveness. Third, a higher effort yields more precise signals, but leads to more disutility. The left-hand side of (5) reflects the benefit. Because both $f_{1}$ and $f_{2}$ are densities, the integral in (5) would have been zero if the lower limit was set to $\underline{s}$. Now by the Informativeness Property, this integral with lower limit at $\widehat{s}^{f b}\left(e^{f b}\right)>\underline{s}$ must be strictly positive, and it measures how strongly higher values of $s$ leads to cost-effective assignments of clients. The right-hand side of (5) is the marginal disutility of effort.

We assume that clients are matched randomly to experts, and pay a fixed tariff, $T$, to the expert who renders a service. Each client obtains the same benefit from an expert and each expert's ex ante cost for treating a random client is equal to the average cost. The only restriction here is that $T$ is at least the ex ante average cost, $\left(c_{L}+c_{H}\right) / 2$. In Section 5, we endogenize the tariff (and also the initial assignment of clients) by letting experts compete in a Bertrand fashion. Our results are unchanged with endogenously chosen tariffs. ${ }^{5}$

${ }^{4}$ Consider $-0.5 \Delta \int_{\widehat{s}}^{\bar{s}}\left\{f_{2}(x \mid e)-f_{1}(x \mid e)\right\} \mathrm{d} x+\phi(e)$, which is the expected cost at threshold $\widehat{s}$ (after some constants have been dropped). The Hessian of this expected cost is:

$$
\left[\begin{array}{cc}
-0.5 \Delta \int_{\widehat{s}}^{\bar{s}}\left\{\frac{\partial^{2} f_{2}(x \mid e)}{\partial e^{2}}-\frac{\partial^{2} f_{1}(x \mid e)}{\partial e^{2}}\right\} \mathrm{d} x+\phi^{\prime \prime}(e) & 0.5 \Delta\left\{\frac{\partial f_{2}(\widehat{s} \mid e)}{\partial e}-\frac{\partial f_{1}(\widehat{s} \mid e)}{\partial e}\right\} \\
0.5 \Delta\left\{\frac{\partial f_{2}(\widehat{s} \mid e)}{\partial e}-\frac{\partial f_{1}(\widehat{s} \mid e)}{\partial e}\right\} & 0.5 \Delta\left\{\frac{\partial f_{2}(\widehat{s} \mid e)}{\partial s}-\frac{\partial f_{1}(\widehat{s} \mid e)}{\partial s}\right\}
\end{array}\right] .
$$

Convexity requires that the Hessian is positive definite.

${ }^{5}$ Any given value of the tariff and initial assignment define a valid subgame of the extensive form to be presented, so our analysis for arbitrary tariff and assignment is necessary even when tariffs are determined endogenously. 


\section{Referral market}

We look for perfect-Bayesian equilibria of the following extensive form:

Stage 0 For each client, his cost type, either $\omega_{1}$ or $\omega_{2}$, is drawn independently with equal probabilities. The draw is never observed by a client or an expert. Half of all clients are matched with Expert 1, and the other half with Expert 2.

Stage 1 An expert decides on an effort for a matched client. Then the expert observes a realization of the signal for each exerted effort. The effort and signal are the expert's private information.

Stage 2 For each client, an expert chooses between keeping the client and referring the client to the other expert at a price that he chooses.

Stage 3 If an expert has received a referral at some price, the expert decides whether to accept the referral or reject it. If the expert accepts the referral, he pays the other expert the referral price, provides service to the client, incurs the cost (as the client's state eventually realizes), and receives the tariff. If he rejects the referral, the referring expert will render service and receive the tariff.

In Stage 3, an expert may not acquire information before deciding between accepting and rejecting a referral. This may be due to an expert having no access to the client until he has accepted the referral. Alternatively, information acquisition may be time-consuming, and delays may be unacceptable to clients. Finally, a model with multiple rounds of information acquisition together with offers and counteroffers, is less tractable, and outside the scope here.

An expert's payoff comes from one of three events. First, if an expert has kept his own client, he gets the tariff, and incurs the service cost and effort disutility. Second, if an expert has accepted a referral, he pays the referral price, keeps the tariff, and incurs the service cost. Third, if an expert's referral has been accepted, he gets the referral price and incurs the effort disutility. Each expert has a reservation utility that is set at 0 . The referral price made by an expert can be positive or negative.

An expert's strategy is defined by (i) an effort in Stage 1, (ii) a referral decision and price in Stage 2 as a function of the expert's own signal, and (iii) a referral-acceptance decision in Stage 3 as a function of the referral price. A perfect-Bayesian equilibrium consists of a pair of strategies that are mutual best responses, and beliefs about (unobserved) effort and signals, which are updated according to strategies and Bayes' rule whenever possible.

There are many unreached information sets. For example, in an equilibrium, Expert 1 may take some effort $e_{1}$, make a referral offer at price $p_{1}$ if and only if signal $s$ is above a certain threshold. What would Expert 2 believe about Expert 1's effort and signal if Expert 1's referral price were $p_{1}^{\prime} \neq p_{1}$ ? Also, in an equilibrium, an expert may not make any referral at all, so all referral prices are off-equilibrium. Perfect-Bayesian equilibria do not impose belief restrictions at out-of-equilibrium information sets. Multiple equilibria can be supported by many off-path beliefs (and will be discussed later). We will impose a natural and simple belief restriction to be defined in Section 3.

We construct an equilibrium with the following outcome: Expert 1 exerts a strictly positive effort, but Expert 2 does not. Expert 1 refers at a price for all signals above a threshold. Expert 2 does not refer. We will begin the construction by presenting necessary conditions, then prove existence by a standard fixed-point argument.

Equilibrium referral and acceptance. Consider any equilibrium in which Expert 1 has taken an effort, say, $e_{1}>0$, and has observed a signal $s$ in Stage 1. In a continuation equilibrium in Stage 2, if Expert 1 makes a referral that will be accepted, Expert 1 must make it at the highest possible price. Thus, Expert 2's equilibrium strategy in Stage 3 must be to reject any referral price higher than a threshold, and accept it otherwise. 
Suppose that Expert 2 accepts a referral at the threshold price $p_{1}$. How should Expert 1 choose between keeping and referring the client? Given that Expert 1 has taken effort $e_{1}$ and observed signal $s$, the expected payoff (net from effort disutility) from keeping the client is

$$
T-\operatorname{Pr}\left(\omega_{1} \mid s, e_{1}\right) c_{L}-\operatorname{Pr}\left(\omega_{2} \mid s, e_{1}\right) c_{H} .
$$

Because this is decreasing in $s$ by MLRP, we conclude that Expert 1 will refer the client with signal $s$ whenever $s>\widehat{s}$ where

$$
T-\operatorname{Pr}\left(\omega_{1} \mid \widehat{s}, e_{1}\right) c_{L}-\operatorname{Pr}\left(\omega_{2} \mid \widehat{s}, e_{1}\right) c_{H}=p_{1} .
$$

Clearly, we can repeat the same steps for Expert 2's referral decision given that Expert 1 accepts a referral if the price is below a threshold. We summarize the result in the following lemma, whose proof is already in the text above.

Lemma 1. In an equilibrium, in Stage 3, an expert's referral is accepted if and only if the referral price is at or below a threshold, and in Stage 2, an expert makes a referral if and only if the signal exceeds a threshold.

Lemma 1 asserts that, in any equilibrium in which effort is positive, referral decisions and acceptance decisions must be threshold policies. Transmission of the private signal from information-acquisition effort must be pooling. We emphasize that the incentive of referring lemons and keeping peaches holds true for both experts - although each expert has a comparative advantage in a different state. ${ }^{6}$ Lemma 1 does not assert uniqueness: there can be multiple equilibria with different Expert 1 referral thresholds and Expert 2 price-acceptance thresholds.

Expert 1's equilibrium referral and effort. We now focus on Expert 1's referral under the assumption that he has taken an effort. First, we introduce a belief restriction:

Definition 1 (passive belief). A perfect-Bayesian equilibrium is said to satisfy passive belief if an expert's belief about the hidden effort and signal on any off-equilibrium referral price remains the same as the belief at the equilibrium referral price.

Passive belief was first introduced by McAfee and Schwartz (1994) in the context of multilateral contracts. ${ }^{7}$ Here, it says that deviations are uncorrelated trembles. Suppose that, in an equilibrium, Expert 1 takes effort $e_{1}$, and makes a referral offer at price $p_{1}$ if and only if $s$ is above a certain threshold. If Expert 2 receives a referral price $p_{1}^{\prime} \neq p_{1}$, passive belief specifies that Expert 2 continues to believe that Expert 1 has taken effort $e_{1}$ and has made a referral because the signal is above the same threshold. The restriction requires Expert 2 to believe that his expected cost remains at the same equilibrium level even when Expert 1 offers an off-equilibrium referral price.

Lemma 2. Under passive belief, in an equilibrium in which Expert 1 takes effort $e_{1}$ and refers whenever $s>\widehat{s}$, Expert 1's equilibrium referral price $p_{1}$ must be:

$$
p_{1}=T-\operatorname{Pr}\left(\omega_{1} \mid s>\widehat{s}, e_{1}\right)\left(c_{L}+\Delta\right)-\operatorname{Pr}\left(\omega_{2} \mid s>\widehat{s}, e_{1}\right)\left(c_{H}-\Delta\right),
$$

so Expert 2's equilibrium expected utility must equal the outside option.

\footnotetext{
${ }^{6}$ Ours is not a cheap-talk game in which different prices can convey different intervals of signals. The referral price is binding, and has to be paid if it is accepted.

7 "Passive belief" is a common assumption in models of foreclosure, delegation, and integrations; see Dequiedt and Martimort (2015), de Fontenay and Gans (2005), Hart and Tirole (1990), Laffont and Martimort (2000), O'Brien and Shaffer (1992), Reisinger and Tarantino (2015), and Rey and Tirole (2007). More recently, it has also been used in the consumer-search literature; see Bar-Isaac, Caruana, and Cunat (2012), Buehler and Schuett (2014), and Inderst and Ottaviani (2012).
} 
The proof of Lemma 2 is this. When Expert 2 receives a referral, according to passive belief, he must believe that Expert 1's signal is above $\widehat{s}$. Expert 2's expected cost of providing service to the referred client is $\operatorname{Pr}\left(\omega_{1} \mid s>\widehat{s}, e_{1}\right)\left(c_{L}+\Delta\right)+\operatorname{Pr}\left(\omega_{2} \mid s>\widehat{s}, e_{1}\right)\left(c_{H}-\Delta\right)$. Therefore, Expert 2 accepts the referral if and only if the price is lower than $T-\operatorname{Pr}\left(\omega_{1} \mid s>\widehat{s}, e_{1}\right)\left(c_{L}+\Delta\right)-\operatorname{Pr}\left(\omega_{2} \mid s>\right.$ $\left.\widehat{s}, e_{1}\right)\left(c_{H}-\Delta\right)$. Given this best response by Expert 2, Expert 1 optimally chooses the highest price that will be accepted. This is the definition of $p_{1}$ in (8). Clearly, Expert 2 earns a zero-expected utility when he accepts a referral. (The equilibrium referral price may be negative; Expert 1 may have to pay Expert 2 in order to cover the expected cost because the tariff is low. For example, if $T$ is just equal to the average of $c_{L}$ and $c_{H}$, then $T$ cannot cover Expert 2's expected cost, but Expert 1 will set $p_{1}$ to be negative to cover that loss. Expert 1 will do this because his loss without a referral would be even higher.)

Passive belief does rule out many other equilibria. In these equilibria, Expert 2 earns strictly more than the outside option, but referral happens less often. To see this, choose $\varepsilon>0$, and for the same effort $e_{1}$ and some signal threshold $\widehat{s}^{\prime}$, consider a referral price $p_{1}^{\prime}$ satisfying

$$
p_{1}^{\prime}+\varepsilon=T-\operatorname{Pr}\left(\omega_{1} \mid s>\widehat{s}^{\prime}, e_{1}\right)\left(c_{L}+\Delta\right)-\operatorname{Pr}\left(\omega_{2} \mid s>\widehat{s}^{\prime}, e_{1}\right)\left(c_{H}-\Delta\right) .
$$

Now Expert 2's strategy is to accept a referral at price $p_{1}^{\prime}$ or lower. Expert 2 believes that the signal is at least $\widehat{s}^{\prime}$. If Expert 1 offers $p_{1}>p_{1}^{\prime}$, Expert 2 would change his belief; he now believes that the signal threshold has increased from $\widehat{s}^{\prime}$ (perhaps all the way to $\bar{s}$ ). Now that the bad state is thought to be more likely, Expert 2's expected cost has increased, so he rejects $p_{1}$. Expert 1 is then stuck with having to refer at a price that leaves some rent. Passive belief rules out such a discontinuous change: when a referral is made at a higher price, Expert 2 must continue to believe that the referral threshold is $\widehat{s}^{\prime}$. (See also the discussion following Proposition 1.) From now on, we will always use passive belief.

We continue to characterize Expert 1's referral threshold $\widehat{s}$. Recall that Expert 1's payoff from keeping a client with signal $s$ is (6). Given that Expert 2 accepts a referral at price $p_{1}$, Expert 1 refers a client with signal $s>\widehat{s}$ if and only if $p_{1}=T-\operatorname{Pr}\left(\omega_{1} \mid \widehat{s}, e_{1}\right) c_{L}-\operatorname{Pr}\left(\omega_{2} \mid \widehat{s}, e_{1}\right) c_{H}$. As an intermediate step, we present a basic property about experts' expected costs conditional on signals. (The proof is in the Appendix.)

Lemma 3. For $e_{1}>0$, the equation

$$
\begin{aligned}
& \operatorname{Pr}\left(\omega_{1} \mid \widehat{s}, e_{1}\right) c_{L}+\operatorname{Pr}\left(\omega_{2} \mid \widehat{s}, e_{1}\right) c_{H} \equiv \frac{c_{L} f_{1}\left(\widehat{s} \mid e_{1}\right)+c_{H} f_{2}\left(\widehat{s} \mid e_{1}\right)}{f_{1}\left(\widehat{s} \mid e_{1}\right)+f_{2}\left(\widehat{s} \mid e_{1}\right)} \\
& =\frac{\left(c_{L}+\Delta\right) \int_{\widehat{s}}^{\bar{s}} f_{1}\left(x \mid e_{1}\right) \mathrm{d} x+\left(c_{H}-\Delta\right) \int_{\widehat{s}}^{\bar{s}} f_{2}\left(x \mid e_{1}\right) \mathrm{d} x}{\int_{\widehat{s}}^{\bar{s}} f_{1}\left(x \mid e_{1}\right) \mathrm{d} x+\int_{\widehat{s}}^{\bar{s}} f_{2}\left(x \mid e_{1}\right) \mathrm{d} x} \\
& \equiv \operatorname{Pr}\left(\omega_{1} \mid s>\widehat{s}, e_{1}\right)\left(c_{L}+\Delta\right)+\operatorname{Pr}\left(\omega_{2} \mid s>\widehat{s}, e_{1}\right)\left(c_{H}-\Delta\right)
\end{aligned}
$$

has a unique solution $\underline{s} \leq \widehat{s} \leq \bar{s}$.

Suppose that Expert 1 has chosen effort $e_{1}$. Expert 1's expected cost at signal $s$ is (9), whereas Expert 2's expected cost conditional on signals above $s$ is (10). The lemma says that there is one and only one signal $\widehat{s}$ at which Expert 1's expected cost at $\widehat{s}$ is the same as Expert 2's expected cost at signals above $\widehat{s}$.

This result stems from Expert 2's comparative advantage in providing services to clients at state $\omega_{2}$. Figure 1 graphs three expected costs. The solid line is Expert 1's expected cost at signal $s$ (9). The dotted line is Expert 1's expected cost conditional on signals above $s$ :

$$
\operatorname{Pr}\left(\omega_{1} \mid s>\widehat{s}, e_{1}\right) c_{L}+\operatorname{Pr}\left(\omega_{2} \mid s>\widehat{s}, e_{1}\right) c_{H} \equiv \frac{c_{L} \int_{\widehat{s}}^{\bar{s}} f_{1}\left(x \mid e_{1}\right) \mathrm{d} x+c_{H} \int_{\widehat{s}}^{\bar{s}} f_{2}\left(x \mid e_{1}\right) \mathrm{d} x}{\int_{\widehat{s}}^{\bar{s}} f_{1}\left(x \mid e_{1}\right) \mathrm{d} x+\int_{\widehat{s}}^{\bar{s}} f_{2}\left(x \mid e_{1}\right) \mathrm{d} x} .
$$




\section{FIGURE 1}

\section{EXPECTED COSTS AND EXPERT 1'S REFERRAL THRESHOLD $\widehat{S}$}

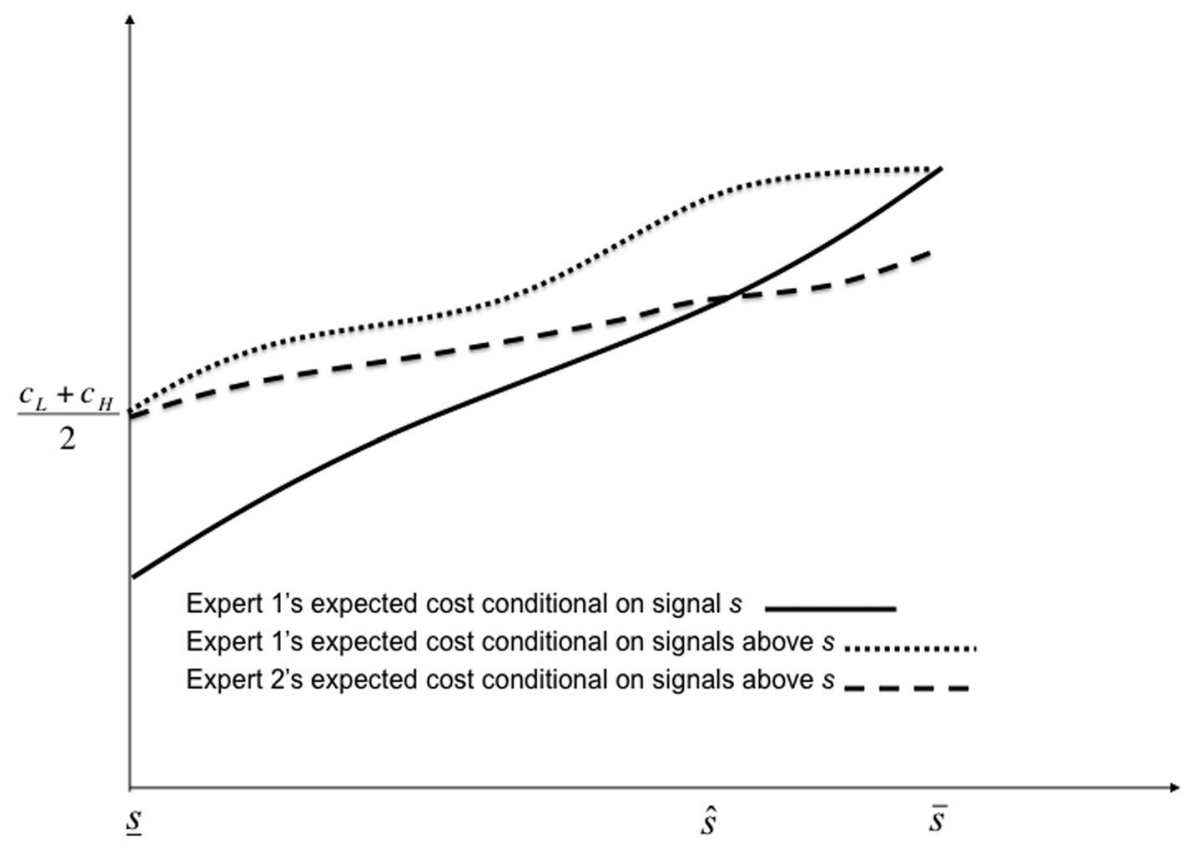

By MLRP, a higher $s$ means that state $\omega_{2}$ is more likely, so Expert 1's cost at $s$ is increasing in $s$. It also means that Expert 1's cost conditional on signals above $s$ is increasing and even higher. However, cost at $s$ and cost conditional on signals above $s$ converge at $s=\bar{s}$.

In Figure 1, the dashed line is Expert 2's expected cost conditional on signals above $s$. Due to Expert 2's comparative advantage, his cost conditional on signals above $s$ is smaller than Expert 1 's. However, this comparative advantage vanishes as the conditioning threshold $s$ drops toward $\underline{s}$. In other words, because conditioning on all signals above $\underline{s}$ means no information, the two experts have the same cost $\left(c_{L}+c_{H}\right) / 2$. It follows that there is a signal $\widehat{s}$ at which Expert 1 's cost at $\widehat{s}$ is equal to Expert 2's cost, conditional on signals above $\widehat{s}$. In the Appendix, we prove uniqueness of $\widehat{s}$ by showing that the slopes of Expert 1's cost at signal $s$ and Expert 2's cost conditional on signals above $s$ cannot be the same.

The significance of Lemma 3 is this. Although Expert 1's referrals pool all clients with signals higher than $\widehat{s}$, the experts nevertheless can mutually benefit from trade due to Expert 2 's cost comparative advantage at state $\omega_{2}$. Expert 1's referrals are all lemons, but Expert 2's has lower expected cost servicing lemons. Given effort $e_{1}$, as long as the signal is above $\widehat{s}$, the one in Lemma 3, a successful referral happens in equilibrium, as the next result shows (the proof is in the Appendix).

Proposition 1. In an equilibrium in which Expert 1 exerts strictly positive effort $e_{1}$, he refers a client with a signal $s \geq \widehat{s}$ to Expert 2 at a price $p_{1}$, and Expert 2 accepts a referral if and only if Expert 1's price is at most $p_{1}$, where

$$
\begin{aligned}
T- & \frac{\left(c_{L}+\Delta\right) \int_{\widehat{s}}^{\bar{s}} f_{1}\left(x \mid e_{1}\right) \mathrm{d} x+\left(c_{H}-\Delta\right) \int_{\widehat{s}}^{\bar{s}} f_{2}\left(x \mid e_{1}\right) \mathrm{d} x}{\int_{\widehat{s}}^{\bar{s}} f_{1}\left(x \mid e_{1}\right) \mathrm{d} x+\int_{\widehat{s}}^{\bar{s}} f_{2}\left(x \mid e_{1}\right) \mathrm{d} x}=p_{1} \\
& =T-\frac{c_{L} f_{1}\left(\widehat{s} \mid e_{1}\right)+c_{H} f_{2}\left(\widehat{s} \mid e_{1}\right)}{f_{1}\left(\widehat{s} \mid e_{1}\right)+f_{2}\left(\widehat{s} \mid e_{1}\right)} .
\end{aligned}
$$


In (12), the first equation says that Expert 2 is indifferent between accepting all referrals of clients with signals above $\widehat{s}$ and rejecting. The second equation says that Expert 1 is indifferent between keeping clients with signal $\widehat{s}$ and referring. Together, they determine the continuation referral equilibrium, given effort $e_{1}$. These are mutual best responses. Proposition 1 stems from classical adverse selection. Expert 1's referral is based on his private information, so client $\widehat{s}$ is his marginal client. Expert 2 faces the average client with signals above $\widehat{s}$. Adverse selection does not rule out trade because of cost comparative advantage.

Again, passive belief does rule out other continuation equilibria. For the same effort $e_{1}$, other equilibria with referral price $p_{1}^{\prime}$ and referral threshold $\widehat{s}^{\prime}$ are possible. Let

$$
\begin{aligned}
T- & \frac{\left(c_{L}+\Delta\right) \int_{\widehat{s}^{\prime}}^{\bar{s}} f_{1}\left(x \mid e_{1}\right) \mathrm{d} x+\left(c_{H}-\Delta\right) \int_{\widehat{s}^{\prime}}^{\bar{s}} f_{2}\left(x \mid e_{1}\right) \mathrm{d} x}{\int_{\widehat{s}^{\prime}}^{s} f_{1}\left(x \mid e_{1}\right) \mathrm{d} x+\int_{\widehat{s}^{\prime}}^{\bar{s}} f_{2}\left(x \mid e_{1}\right) \mathrm{d} x}-\varepsilon=p_{1}^{\prime} \\
& =T-\frac{c_{L} f_{1}\left(\widehat{s}^{\prime} \mid e_{1}\right)+c_{H} f_{2}\left(\widehat{s}^{\prime} \mid e_{1}\right)}{f_{1}\left(\widehat{s}^{\prime} \mid e_{1}\right)+f_{2}\left(\widehat{s}^{\prime} \mid e_{1}\right)} .
\end{aligned}
$$

Here, Expert 1's referral price is $p_{1}^{\prime}$, and Expert 2's equilibrium payoff is at $\varepsilon>0$. If Expert 1 raised the price from $p_{1}^{\prime}$ to extract more rent, Expert 2 now would believe that the client had a very high signal, say $\bar{s}$, and would reject the higher price. This continuation equilibrium is consistent with Lemma 1, but violates passive belief. Under passive belief, any price between $p_{1}^{\prime}$ and $p_{1}^{\prime}+\varepsilon$ must be accepted by Expert 2. For effort $e_{1}$, the continuation equilibrium in Proposition 1 has the most referrals.

We next study Expert 1's effort incentive. If $e_{1}$ is an equilibrium effort, given that Expert 2 will accept a referral at price $p_{1}$, Expert 1's referral threshold is in (7). Recalling that the ex ante density of $s$ is $0.5\left[f_{1}\left(s \mid e_{1}\right)+f_{2}\left(s \mid e_{1}\right)\right]$, we write Expert 1's expected payoff per client as

$$
\begin{aligned}
& \int_{\underline{s}}^{\widehat{s}} 0.5\left[\left(T-c_{L}\right) \operatorname{Pr}\left(\omega_{1} \mid x, e_{1}\right)+\left(T-c_{H}\right) \operatorname{Pr}\left(\omega_{2} \mid x, e_{1}\right)\right]\left[f_{1}\left(x \mid e_{1}\right)+f_{2}\left(x \mid e_{1}\right)\right] \mathrm{d} x \\
& \quad+p_{1} \int_{\widehat{s}}^{\bar{s}} 0.5\left[f_{1}\left(x \mid e_{1}\right)+f_{2}\left(x \mid e_{1}\right)\right] \mathrm{d} x-\phi\left(e_{1}\right) .
\end{aligned}
$$

From the definition of $\widehat{s}$ in (7), the first integral above is Expert 1's expected utility when he keeps the client ( $s$ below $\widehat{s}$ ), whereas the second is the expected utility when he successfully refers ( $s$ above $\widehat{s}$ ). Using the expressions for the conditional probabilities of the states $\omega_{1}$ and $\omega_{2}$, we simplify the payoff per client to

$$
\left.\left[T-\frac{c_{L}+c_{H}}{2}\right]-\phi\left(e_{1}\right)+0.5 \int_{\widehat{s}}^{\bar{s}}\left\{\left[p_{1}-\left(T-c_{L}\right)\right] f_{1}\left(x \mid e_{1}\right)+\left[p_{1}-\left(T-c_{H}\right)\right] f_{2}\left(x \mid e_{1}\right)\right]\right\} \mathrm{d} x .
$$

The first term in (13) is the expected payoff from treating a randomly chosen client; effort has a cost, the second term, but generates an expected benefit, the difference between the referral price $p_{1}$ and what Expert 1 would have obtained if he had kept the client (the integral).

In an equilibrium in which Expert 1's effort is positive, his equilibrium effort $e_{1}^{*}$ maximizes (13) where the threshold $\widehat{s}$ is given by (7). The first-order condition characterizes Expert 1 's equilibrium effort:

$$
0.5 \int_{\widehat{s}}^{\bar{s}}\left\{\left[p_{1}-\left(T-c_{L}\right)\right] \frac{\partial f_{1}\left(x \mid e_{1}\right)}{\partial e_{1}}+\left[p_{1}-\left(T-c_{H}\right)\right] \frac{\partial f_{2}\left(x \mid e_{1}\right)}{\partial e_{1}}\right\} \mathrm{d} x=\phi^{\prime}\left(e_{1}\right) .
$$

By the Informativeness Property, $\int_{\widehat{s}}^{\bar{s}} \frac{\partial f_{1}\left(x \mid e_{1}\right)}{\partial e_{1}} \mathrm{~d} x<0<\int_{\widehat{s}}^{\bar{s}} \frac{\partial f_{2}\left(x \mid e_{1}\right)}{\partial e_{1}} \mathrm{~d} x$. Also, because $c_{H}>c_{L}$, for any $p_{1}$ between $T-c_{H}$ and $T-c_{L}$, the term inside the curly brackets in (14) must be strictly positive. Therefore, equation (14) is consistent with a strictly positive equilibrium effort. 
Expert 2's equilibrium effort. We now turn to Expert 2's equilibrium effort and referrals. Indeed, one might have thought that some "symmetry" might apply so Expert 2 could exploit cost comparative advantage. The answer is negative, as stated in the next proposition (the proof is in the Appendix).

Proposition 2. In any equilibrium, Expert 2 does not exert any effort or make any referral.

Expert 1 has cost comparative advantage in the good state $\omega_{1}$, so if there was any referral to exploit that advantage, Expert 2 would have to refer clients with low signals. Lemma 1, however, says that an expert would like to keep only clients with low signals; an expert will never refer peaches, only lemons. If Expert 1 believed that Expert 2 was referring clients with low signals, Expert 2 would cheat and refer clients with high signals. However, for clients with signals above a threshold, Expert 1's expected costs will never be lower than Expert 2's. There is no possibility of mutually beneficial trade. Given that in equilibrium Expert 2 does not refer, there is no incentive for him to acquire information.

Because Expert 2 does not make any equilibrium referral, all referral price offers to Expert 1 are off-equilibrium, so passive belief has no bite. For later use, we note that the highest posterior belief on the bad state $\omega_{2}$ can be written as $\operatorname{Pr}\left(\omega_{2} \mid \bar{s}, \widetilde{e}\right)$ where $\widetilde{e}=\operatorname{argmax}_{e} f_{2}(\bar{s} \mid e) / f_{1}(\bar{s} \mid e)$. We say that an expert has the most pessimistic belief if he believes that the other expert has taken effort $\widetilde{e}$ and has observed the signal $\bar{s}$.

Equilibrium information acquisition and referral. Now we put together our earlier results and state the following (the proof is in the Appendix).

Proposition 3. There is an equilibrium characterized by the triple $\left[e_{1}^{*}, \widehat{s}^{*}, p_{1}^{*}\right]$ such that (i) $\left(e_{1}^{*}, \widehat{s}^{*}\right)$ maximize (13) given $p_{1}^{*}$, and (ii) $p_{1}^{*}$ is given by (12) at $\widehat{s}^{*}$ and $e_{1}^{*}$. The equilibrium strategies and beliefs are:

(a) Expert 1 chooses effort $e_{1}^{*}$ and refers a client with any signal $s>\widehat{s}^{*}$ at price $p_{1}^{*}$ and keeps other clients.

(b) Expert 2 chooses zero effort, does not refer, and accepts a referral if and only if the referral price is at most $p_{1}^{*}$.

(c) If Expert 2 receives a referral at a price different from $p_{1}^{*}$, he continues to believe that Expert 1 has chosen effort $e_{1}^{*}$ and referred a client with signal $s>\widehat{s}^{*}$.

(d) If Expert 1 receives a referral offer from Expert 2 at any price, Expert 1 has the most pessimistic belief (he believes that Expert 2's effort is $\widetilde{e}$ and his signal is $\bar{s}$, where $\widetilde{e}=$ $\left.\operatorname{argmax}_{e} f_{2}(\bar{s} \mid e) / f_{1}(\bar{s} \mid e)\right)$.

In Proposition 3, the first three points in the strategy and belief description follow directly from the results above. The fourth point is about Expert 1's response against off-equilibrium referral prices. We specify that Expert 1 has the most pessimistic belief. This deters Expert 2 from deviating to a positive effort, and referring a client when the signal indicates an expected loss. If Expert 1 believes that Expert 2 has taken no effort, he will accept any offer $p$ when $T-\left(c_{L}+c_{H}\right) / 2-p \geq 0$. Now if Expert 2 chooses effort $e_{2}>0$, and he observes an $s$ where his expected cost is higher than the ex ante cost: $\operatorname{Pr}\left(\omega_{1} \mid s, e_{2}\right)\left(c_{L}+\Delta\right)+\operatorname{Pr}\left(\omega_{2} \mid s, e_{2}\right)\left(c_{H}-\Delta\right)>$ $\left(c_{L}+c_{H}\right) / 2$, Expert 2 will profit by successfully referring at $p=T-\left(c_{L}+c_{H}\right) / 2$. Of course, this is inconsistent with Proposition 2, so Expert 1 believing Expert 2 having taken zero effort cannot be part of off-equilibrium belief. To support the equilibrium, we have chosen the most pessimistic off-equilibrium belief when no price is ever offered in equilibrium, and show in the proof that Expert 2 has no profitable deviation from zero effort. The final step in the proof is a standard, fixed-point argument for the existence of $\left[e_{1}^{*}, \widehat{s}^{*}, p_{1}^{*}\right]$. 
Expert 2 does not exert any effort, which, of course, is inefficient. What about Expert 1's equilibrium effort and referrals? Using Proposition 1, and the first-order condition for Expert 1's equilibrium effort, we write down the conditions for the referral equilibrium $\left[e_{1}^{*}, \widehat{s}^{*}, p_{1}^{*}\right]$ :

$$
\begin{gathered}
T-\frac{\left(c_{L}+\Delta\right) \int_{\widehat{S}^{*}}^{\bar{s}} f_{1}\left(x \mid e_{1}^{*}\right) \mathrm{d} x+\left(c_{H}-\Delta\right) \int_{\widehat{S}^{*}}^{\bar{s}} f_{2}\left(x \mid e_{1}^{*}\right) \mathrm{d} x}{\int_{\widehat{s}^{*}}^{\bar{s}} f_{1}\left(x \mid e_{1}^{*}\right) \mathrm{d} x+\int_{\widehat{s}^{*}}^{\bar{s}} f_{2}\left(x \mid e_{1}^{*}\right) \mathrm{d} x}=p_{1}^{*} \\
=T-\frac{c_{L} f_{1}\left(\widehat{s}^{*} \mid e_{1}^{*}\right)+c_{H} f_{2}\left(\widehat{s}^{*} \mid e_{1}^{*}\right)}{f_{1}\left(\widehat{s}^{*} \mid e_{1}^{*}\right)+f_{2}\left(\widehat{s}^{*} \mid e_{1}^{*}\right)} \\
0.5 \int_{\widehat{s}^{*}}^{\bar{s}}\left\{\left[p_{1}^{*}-\left(T-c_{L}\right)\right] \frac{\partial f_{1}\left(x \mid e_{1}^{*}\right)}{\partial e_{1}}+\left[p_{1}^{*}-\left(T-c_{H}\right)\right] \frac{\partial f_{2}\left(x \mid e_{1}^{*}\right)}{\partial e_{1}}\right\} \mathrm{d} x=\phi^{\prime}\left(e_{1}^{*}\right) .
\end{gathered}
$$

Proposition 4. In an equilibrium, Expert 1's effort and referral threshold cannot be first best. Furthermore, given equilibrium effort $e_{1}^{*}$, Expert 1's referral threshold $\widehat{s}^{*}$ is too high, $f_{2}\left(\widehat{s}^{*} \mid e_{1}^{*}\right)>$ $f_{1}\left(\widehat{s}^{*} \mid e_{1}^{*}\right)$, so Expert 1 sometimes keeps a client even when his expected service cost is higher than Expert 2's.

At the equilibrium referral threshold, Expert 1's expected cost at $\widehat{s}^{*}$ equals Expert 2's expected cost when signals are all above $\widehat{s}^{*}$. Given $\Delta>0$ and MLRP, equality of the Expert 1 's "marginal" cost and Expert 2's "average" cost requires $f_{2}\left(\widehat{s}^{*} \mid e_{1}^{*}\right)>f_{1}\left(\widehat{s}^{*} \mid e_{1}^{*}\right)$. (See also the proof of Proposition 4 in the Appendix.)

What about Expert 1's equilibrium effort? Using (15), we rewrite (16) as

$$
0.5\left[\frac{c_{H}-c_{L}}{2}\right] \int_{\widehat{s}^{*}}^{\bar{s}}\left\{\frac{2 f_{1}\left(\widehat{s}^{*} \mid e_{1}^{*}\right) \frac{\partial f_{2}\left(x \mid e_{1}^{*}\right)}{\partial e_{1}}-2 f_{2}\left(\widehat{s}^{*} \mid e_{1}^{*}\right) \frac{\partial f_{1}\left(x \mid e_{1}^{*}\right)}{\partial e_{1}}}{f_{1}\left(\widehat{s}^{*} \mid e_{1}^{*}\right)+f_{2}\left(\widehat{s}^{*} \mid e_{1}^{*}\right)}\right\} \mathrm{d} x=\phi^{\prime}\left(e_{1}^{*}\right) .
$$

The left-hand side of this expression is the marginal benefit of effort. There are two effects. First, because $c_{H}-c_{L}>2 \Delta$, so compared to the first best, the cost differential $c_{H}-c_{L}$ affects the marginal benefit more strongly than the cost saving $\Delta$. Second, we have $\widehat{s}^{*}$ strictly higher than the value at the cost-effective threshold (where $f_{2}\left(s \mid e_{1}^{*}\right)=f_{1}\left(s \mid e_{1}^{*}\right)$ ), so the range of the integral is smaller. Moreover, because $f_{2}\left(\widehat{s}^{*} \mid e_{1}^{*}\right)>f_{1}\left(\widehat{s}^{*} \mid e_{1}^{*}\right)$, the weight on the partial derivative $\partial f_{2} / \partial e_{1}$ is smaller than 1, whereas the weight on $\partial f_{1} / \partial e_{1}$ is larger than 1. (By the Informativeness Property, we have $\int_{\widehat{s}^{*}}^{\bar{s}} \frac{\partial f_{1}\left(x \mid e_{1}^{*}\right)}{\partial e_{1}} \mathrm{~d} x<0$, whereas, $\int_{\widehat{s}^{*}}^{\bar{s}} \frac{\partial f_{2}\left(x \mid e_{1}^{*}\right)}{\partial e_{1}} \mathrm{~d} x>0$.) Therefore, the overall effect within the integral reduces the marginal benefit. In sum, the equilibrium effort may be smaller or larger than the first best. ${ }^{8}$

Finally, we remark that the characterization of the referral equilibrium in (15) and (16) does not prove uniqueness. Although examples that we have constructed so far have all produced a unique equilibrium, we have not proven it. Formally, Lemma 3 does show that for any given effort, (15) admits a unique solution for the price and referral threshold. It remains possible, however, that (16) admits multiple solutions in effort, but our characterization applies to every equilibrium.

\section{Organizations}

Equilibria in the referral market are inefficient. An expert organization can perform better. As we have hypothesized in the Introduction, the key difference between an open market and an organization is that service costs ex post become verifiable within an organization. The reassignment of cost responsibility is possible. We present a definition:

\footnotetext{
${ }^{8}$ In fact, the proof in the Appendix shows that even if Expert 1 referred a client to Expert 2 if and only if the signal indicated the bad state to be more likely, Expert 1's effort would still be excessive.
} 
Definition 2 (Cost-transfer protocol). Referrals are said to follow the cost-transfer protocol when the referring expert bears the client's cost when service is provided by the referred expert.

The cost-transfer protocol allows an organization to solve the adverse selection problem by transferring costs between experts. When an expert within an organization refers a client to a fellow expert, he is to be held responsible for the costs to be incurred by the referred expert. In other words, an expert fully internalizes the cost consequence of referring the client to another expert.

Using the cost-transfer protocol, many organizations, such as integration and partnership, can achieve the first best. Expert 1 buys out Expert 2, becomes the owner, performs all information acquisition, and refers clients whose signals indicate a higher likelihood of the bad state. Expert 2 becomes an employee, and any cost incurred will be the firm's responsibility. Obviously, Expert 2 buying out Expert 1 achieves the same. Alternatively, the experts can form a partnership. Here, each expert will acquire information, and refers efficiently. The partnership contract specifies that an expert making a referral fully reimburses the service expense.

The (simplistic) solution relies on an expert doing nothing other than providing service at a predetermined set of costs. We now consider a richer environment in which an expert's service includes an additional input: he also supplies an effort that may reduce costs. Cost responsibility implies an incentive of cost reduction. However, the cost-transfer protocol in organizations such as integration and partnership will mute this incentive. We will demonstrate a trade-off between referral efficiency and cost efficiency, but first we extend the basic model to include cost reduction.

Cost-reduction effort. We enrich the model in Section 2 with general cost reduction. First, a client's service cost is now randomly distributed on a positive support $[\underline{c}, \bar{c}]$. Next, each expert has a second hidden action: a cost-reduction effort $r \geq 0$ (besides the information-acquisition effort). Then we define four distributions $G_{i}^{j}$ on $[\underline{c}, \bar{c}]$, where $G_{i}^{j}$ is the distribution of Expert $j$ 's service cost in state $\omega_{i}, i, j=1,2$. The following table defines experts' expected costs across states and at effort $r$ :

\begin{tabular}{lcc}
\hline & State $\omega_{1}$ & State $\omega_{2}$ \\
\hline Expert 1's expected cost & $\int_{\underline{c}}^{\bar{c}} c \mathrm{~d} G_{1}^{1}(c \mid r)=c_{L}-r$ & $\int_{\underline{c}}^{\bar{c}} c \mathrm{~d} G_{2}^{1}(c \mid r)=c_{H}-r$ \\
Expert 2's expected cost & $\int_{\underline{c}}^{\bar{c}} c \mathrm{~d} G_{1}^{2}(c \mid r)=c_{L}+\Delta-r$ & $\int_{\underline{c}}^{\bar{c}} c \mathrm{~d} G_{2}^{2}(c \mid r)=c_{H}-\Delta-r$ \\
\hline
\end{tabular}

so $G_{i}^{j}(\cdot \mid r)$ is Expert $j$ 's cost distribution at effort $r$ and state $i$, and $c_{L}, c_{L}+\Delta, c_{H}-\Delta$, and $c_{H}$ are expected costs at zero cost effort. The effort $r$ reduces each expert's expected cost by $r$ in each state. ${ }^{9}$ An expert incurs a disutility $\psi(r)$ from effort $r$, and the function $\psi$ is increasing and convex, with $\lim _{r \rightarrow 0} \psi^{\prime}(r)=0$, and $\lim _{r \rightarrow \Delta} \psi^{\prime}(r)=+\infty$. The cost effort and its disutility have the usual interpretation: task management, work hours, attention, etc. The last assumptions of the Inada sort ensure that cost comparative advantage is always valid because expected cost reduction never exceeds $\Delta$. The cost effort is to be taken when an expert provides service. We define the efficient cost effort by $r^{*} \equiv \operatorname{argmax}_{r}[r-\psi(r)]$, and the first-best net cost reduction $\gamma \equiv r^{*}-\psi\left(r^{*}\right)$.

Cost-reduction and information-acquisition efforts are orthogonal in the first best and in the market. When each expert is fully responsible for service cost, he chooses effort $r^{*}$, which results in the cost saving $\gamma$. In the first-best, and the market-referral model, both experts take cost effort $r^{*}$, so we simply redefine $c_{L}, c_{L}+\Delta, c_{H}-\Delta$, and $c_{H}$ by lowering each by $\gamma$. The first best and equilibria now refer to these redefined values. Characterizations of equilibrium information effort

${ }^{9}$ We can make the cost reductions different across states. This adds nothing conceptually, but burdens with more notation. 
and referral remain the same. More important, equilibria derived earlier do not depend on cost saving, $\gamma \cdot{ }^{10}$

Cost comparative advantage versus cost effort. In the enriched model, we use the fullsupport cost distribution assumption. An expert takes effort $r^{*}$ if and only if he is fully responsible for costs. Contracts for the efficient cost effort by exploiting shifting cost-distribution supports are infeasible. ${ }^{11}$ In principle, organizations can employ partial cost-sharing contracts (the referring expert, for example, being responsible for $50 \%$ of cost) so that efforts between 0 and $r^{*}$ can be implemented. For brevity, we do not consider partial cost-sharing contracts because they would not change economic principles. In other words, we continue to adopt the cost-transfer protocol: all service costs are to be borne by the referring expert.

The cost-transfer protocol, defined above, eliminates adverse selection, but it also eliminates the cost effort. The referred expert will not take effort to realize the net cost saving $\gamma$. This is the main trade-off. Now we adopt the accounting convention that the tariff stays with the expert who initiates the referral, so under cost-transfer protocol all referrals will be accepted with a zero price. We continue with the assumption that half of all clients are matched with one expert - although the two experts operate within an organization. Consider Expert 1, and suppose that he has taken information-acquisition effort $e_{1}$, and receives a signal $s$ on a client. His own expected service cost is $\operatorname{Pr}\left(\omega_{1} \mid s, e_{1}\right)\left(c_{L}-\gamma\right)+\operatorname{Pr}\left(\omega_{2} \mid s, e_{1}\right)\left(c_{H}-\gamma\right)$ because he chooses cost effort $r^{*}$. Upon a referral, Expert 2 is not responsible for service cost, so he takes zero cost effort. From Expert 1's perspective, if the client is referred to Expert 2, Expert 1 pays a service cost $\operatorname{Pr}\left(\omega_{1} \mid s, e_{1}\right)\left(c_{L}+\Delta\right)-\operatorname{Pr}\left(\omega_{2} \mid s, e_{1}\right)\left(c_{H}-\Delta\right)$. Clearly, Expert 1 refers the client if and only if doing so saves cost or if signal $s$ is larger than $\widetilde{s}_{1}$ defined by

$$
f_{1}\left(\widetilde{s}_{1} \mid e_{1}\right)\left(c_{L}-\gamma\right)+f_{2}\left(\widetilde{s}_{1} \mid e_{1}\right)\left(c_{H}-\gamma\right)=f_{1}\left(\widetilde{s}_{1} \mid e_{1}\right)\left(c_{L}+\Delta\right)+f_{2}\left(\widetilde{s}_{1} \mid e_{1}\right)\left(c_{H}-\Delta\right) .
$$

Simplifying (18), we obtain the following (the proof is in the Appendix):

Lemma 4. At effort $e_{1}$, Expert 1 refers a client to Expert 2 if and only if signal $s$ is higher than $\tilde{s}_{1}$, where

$$
\frac{f_{1}\left(\widetilde{s}_{1} \mid e_{1}\right)}{f_{2}\left(\widetilde{s}_{1} \mid e_{1}\right)}=\frac{\Delta-\gamma}{\Delta+\gamma} \leq 1
$$

The threshold $\widetilde{s}_{1}$ is first best at $\gamma=0$, and increases to $\bar{s}$ as $\gamma$ increases to $\Delta$.

Expert 1 will take cost effort $r^{*}$ for his clients to lower his expected cost by $\gamma$, but under the cost-transfer protocol, Expert 2 will not. If a signal indicates that Expert 2's expected cost is lower, it must be because the bad state is much more likely than $1 / 2$. Furthermore, as the net saving from cost effort $\gamma$ increases, cost comparative advantage becomes less important, so referrals become less likely.

From Lemma 4, Expert 1's total expected cost from effort $e_{1}$ is

$$
0.5\left\{\begin{array}{l}
\int_{\underline{s}}^{\widetilde{s}_{1}}\left[f_{1}\left(x \mid e_{1}\right)\left(c_{L}-\gamma\right)+f_{2}\left(x \mid e_{1}\right)\left(c_{H}-\gamma\right)\right] \mathrm{d} x+ \\
\int_{\widetilde{s}_{1}}^{\bar{s}}\left[f_{1}\left(x \mid e_{1}\right)\left(c_{L}+\Delta\right)+f_{2}\left(x \mid e_{1}\right)\left(c_{H}-\Delta\right)\right] \mathrm{d} x
\end{array}\right\}+\phi\left(e_{1}\right) .
$$

Expert 1's payoff is not aligned with the social return to information-acquisition effort. In the first best, Expert 2 chooses cost effort $r^{*}$, but, in an organization, Expert 2 chooses zero cost effort. We present the following result (the proof is in the Appendix):

${ }^{10}$ The key Lemma 3 is unaffected because if each of $c_{L}$ and $c_{H}$ is reduced by $\gamma$, the solution to the equation there remains the same. The value of the equilibrium price will also be reduced by $\gamma$, so the equilibrium effort remains the same.

${ }^{11}$ Suppose the cost distribution support for effort $r^{*}$ is $[\underline{c}, \bar{c}]$, but the support for another effort $r^{\prime}$ is $\left[\underline{c}^{\prime}, \bar{c}^{\prime}\right] \neq[\underline{c}, \bar{c}]$. An expert is deterred from taking effort $r^{\prime}$ if a penalty is imposed whenever the realized cost is not in $\left[c_{1}, c_{2}\right]$ but in $\left[\underline{c}^{\prime}, \bar{c}^{\prime}\right]$. The full support assumption rules out $\left[\underline{c}^{\prime}, \bar{c}^{\prime}\right] \neq[\underline{c}, \bar{c}]$. 
Lemma 5. Expert 1 does not choose the first-best information-acquisition effort in the costtransfer protocol except at $\gamma=0$. As $\gamma$ increases to $\Delta$, Expert 1's information-acquisition effort decreases to 0 .

Information-acquisition effort is beneficial only if it leads to referrals. When cost effort is ineffective $(\gamma=0)$, Expert 1 chooses the first-best information effort because he internalizes cost comparative advantage. As $\gamma$ increases, cost reduction becomes more important, and Expert 1's ex ante expected cost becomes lower, so he refers less often. In the limit when $\gamma=\Delta$, Expert 1 does not acquire information.

Lemmas 4 and 5 hold in a symmetric fashion for Expert 2. We now state these results (but omit their proofs).

Lemma 6. At effort $e_{2}$, Expert 2 refers a client to Expert 1 if and only if signal $s$ is lower than $\widetilde{s}_{2}$, where

$$
\frac{f_{1}\left(\widetilde{s}_{2} \mid e_{2}\right)}{f_{2}\left(\widetilde{s}_{2} \mid e_{2}\right)}=\frac{\Delta+\gamma}{\Delta-\gamma} \geq 1 .
$$

The threshold $\widetilde{s}_{2}$ is first best at $\gamma=0$, and decreases to $\underline{s}$ as $\gamma$ increases to $\Delta$.

Analogous to (20), Expert 2's expected cost from effort $e_{2}$ is

$$
0.5\left\{\begin{array}{c}
\int_{\underline{s}}^{\widetilde{s}_{2}}\left[f_{1}\left(x \mid e_{2}\right) c_{L}+f_{2}\left(x \mid e_{2}\right) c_{H}\right] \mathrm{d} x+ \\
\int_{\widetilde{s}_{2}}^{\bar{s}}\left[f_{1}\left(x \mid e_{2}\right)\left(c_{L}+\Delta-\gamma\right)+f_{2}\left(x \mid e_{2}\right)\left(c_{H}-\Delta-\gamma\right)\right] \mathrm{d} x
\end{array}\right\}+\phi\left(e_{2}\right) .
$$

Lemma 7. Expert 2 does not choose the first-best information-acquisition effort in the costtransfer protocol except at $\gamma=0$. As $\gamma$ increases to $\Delta$, Expert 2's information-acquisition effort decreases to 0 .

Comparison between organization and market. Let $\widetilde{e}_{1}$ and $\widetilde{e}_{2}$ be Expert 1's and Expert 2 's information efforts in the cost-transfer protocol. Expert 1 provides service to clients with signals below $\widetilde{s}_{1}$, and refers those with signals above. Expert 2 provides service to clients with signals above $\widetilde{s}_{2}$, and refers those with signals below. Referrals are always accepted, but the expert who receives a referral will take zero cost effort. The total equilibrium expected costs per client is

$$
\begin{aligned}
& 0.5\left\{\begin{array}{c}
0.5 \int_{\underline{s}}^{\widetilde{s}_{1}}\left[f_{1}\left(x \mid \widetilde{e}_{1}\right)\left(c_{L}-\gamma\right)+f_{2}\left(x \mid \widetilde{e}_{1}\right)\left(c_{H}-\gamma\right)\right] \mathrm{d} x \\
+0.5 \int_{\widetilde{s}_{1}}^{\bar{s}}\left[f_{1}\left(x \mid \widetilde{e}_{1}\right)\left(c_{L}+\Delta\right)+f_{2}\left(x \mid \widetilde{e}_{1}\right)\left(c_{H}-\Delta\right)\right] \mathrm{d} x+\phi\left(\widetilde{e}_{1}\right)
\end{array}\right\} \\
& +0.5\left\{\begin{array}{c}
0.5 \int_{\underline{s}}^{\widetilde{s_{2}}}\left[f_{1}\left(x \mid \widetilde{e}_{2}\right) c_{L}+f_{2}\left(x \mid \widetilde{e}_{2}\right) c_{H}\right] \mathrm{d} x \\
+0.5 \int_{\widetilde{s}_{2}}^{\bar{s}}\left[f_{1}\left(x \mid \widetilde{e}_{2}\right)\left(c_{L}+\Delta-\gamma\right)+f_{2}(x \mid \widetilde{e})\left(c_{H}-\Delta-\gamma\right)\right] \mathrm{d} x+\phi\left(\widetilde{e}_{2}\right)
\end{array}\right\} .
\end{aligned}
$$

These four integrals correspond to different cases of experts retaining and referring clients. We simplify the expected cost per client in the cost-transfer protocol to

$$
\begin{gathered}
\left\{\frac{c_{L}+c_{H}}{2}\right\}-0.5\left\{\gamma \int_{\underline{s}}^{\widetilde{s}_{1}}\left[f_{1}\left(x \mid \widetilde{e}_{1}\right)+f_{2}\left(x \mid \widetilde{e}_{1}\right)\right] \mathrm{d} x+\Delta \int_{\widetilde{s}_{1}}^{\bar{s}}\left[f_{2}\left(x \mid \widetilde{e}_{1}\right)-f_{1}\left(x \mid \widetilde{e}_{1}\right)\right] \mathrm{d} x\right\} \\
-0.5\left\{\gamma \int_{\widetilde{s}_{2}}^{\bar{s}}\left[f_{1}\left(x \mid \widetilde{e}_{2}\right)+f_{2}\left(x \mid \widetilde{e}_{2}\right)\right] \mathrm{d} x+\Delta \int_{\widetilde{s}_{2}}^{\bar{s}}\left[f_{2}\left(x \mid \widetilde{e}_{2}\right)-f_{1}(x \mid \widetilde{e})\right] \mathrm{d} x\right\} \\
+0.5\left[\phi\left(\widetilde{e}_{1}\right)+\phi\left(\widetilde{e}_{2}\right)\right] \equiv E C_{t}(\gamma) .
\end{gathered}
$$


Next, consider a market equilibrium. ${ }^{12}$ Recall from Section 3 that the equilibrium allocation is given by Expert 1's effort $e_{1}^{*}$ and referral threshold $\widehat{s}^{*}$, and Expert 2's zero effort and lack of referral. Each expert uses the first-best cost effort for a $\gamma$ net cost reduction. The total expected cost per client in the equilibrium is

$$
0.5\left\{\begin{array}{c}
\int_{\underline{s}}^{\widehat{s}^{*}}\left[f_{1}\left(x \mid e_{1}^{*}\right) c_{L}+f_{2}\left(x \mid e_{1}^{*}\right) c_{H}\right] \mathrm{d} x \\
+\int_{\widehat{s}^{*}}^{\bar{s}}\left[f_{1}\left(x \mid e_{1}^{*}\right)\left(c_{L}+\Delta\right)+f_{2}\left(x \mid e_{1}^{*}\right)\left(c_{H}-\Delta\right)\right] \mathrm{d} x+\phi\left(e_{1}^{*}\right)
\end{array}\right\}+0.5\left\{\frac{c_{L}+c_{H}}{2}\right\}-\gamma .
$$

Here, each expert takes cost effort, so the net cost saving $\gamma$ applies to each client. Expert 1 takes equilibrium effort $e_{1}^{*}$, and obtains a signal. The first integral is the expected cost of Expert 1's clients with signals below the equilibrium threshold $\widehat{s}^{*}$, and the second integral is the expected cost of Expert 1's referred clients. Expert 2 neither takes effort nor refers in equilibrium, so his expected cost is one half of the sum of $c_{L}$ and $c_{H}$. We simplify the total equilibrium expected cost in the market equilibrium to

$$
\left\{\frac{c_{L}+c_{H}}{2}\right\}-0.5\left\{\Delta \int_{\widehat{S}^{*}}^{\bar{s}}\left[f_{2}\left(x \mid e_{1}^{*}\right)-f_{1}\left(x \mid e_{1}^{*}\right)\right] \mathrm{d} x-\phi\left(e_{1}^{*}\right)\right\}-\gamma \equiv E C_{m}(\gamma) .
$$

Finally, from Section 2, we subtract $\gamma$ from (4) evaluated at the first-best effort to obtain the expected cost under the first best, and we call this $E C_{f b}(\gamma)$. The following presents the trade-off between the market and the expert organization under cost-transfer protocol (the proof is in the Appendix).

Proposition 5. The expected cost per client is lower under cost-transfer protocol than in a market equilibrium if and only if the net cost saving $\gamma$ is below a threshold $\widehat{\gamma}, 0<\widehat{\gamma}<\Delta$.

When net cost saving $\gamma$ vanishes, the cost-transfer protocol achieves the first best. At $\gamma=0$, we have $E C_{t}(\gamma)=E C_{f b}(\gamma)$. The market equilibrium never achieves the first best. However, the market equilibrium always achieves $\gamma$ cost saving because each expert bears his own costs. As $\gamma$ increases from 0 , expected costs in the first best, market, and cost-transfer protocol fall. Both $E C_{f b}(\gamma)$ and $E C_{m}(\gamma)$ fall at a unit rate as $\gamma$ increases. What about the expected cost $E C_{t}(\gamma)$ ? As $\gamma$ increases, referrals become less often under cost-transfer protocol, and information effort becomes less important (see the last four lemmas). As a result, $E C_{t}(\gamma)$ falls at a rate less than 1 as $\gamma$ increases. Beyond a critical value, expected cost of the market equilibrium becomes lower than cost-transfer protocol. The critical value $\widehat{\gamma}$ is obtained by the solution of $E C_{m}(\widehat{\gamma})=E C_{t}(\widehat{\gamma})$.

We illustrate the three expected costs $E C_{m}(\gamma), E C_{t}(\gamma)$, and $E C_{f b}(\gamma)$ in Figure 2. The firstbest cost $E C_{f b}(\gamma)$ is a parallel downward shift of the cost in the market $E C_{m}(\gamma)$. The cost $E C_{t}(\gamma)$ in the expert organization is at the first-best level at $\gamma=0$, but decreases less steeply than the other two.

The basic economics principle is this. In the market, experts work hard to reduce their own costs, but an expert acquires private information, so referrals are subject to adverse selection. In an organization, an expert works hard only if he is responsible for the cost, but cost-transfer protocol avoids asymmetric information. According to Proposition 5, cost-transfer protocol in an organization performs better than the market if and only if cost comparative advantage is more important than cost saving by effort.

Perspectives on legal and medical organizations. Our theory offers a new perspectivetrade-off between adverse selection in the market and shirking within an organization. Proposition 5 predicts that experts form professional organizations when the cost comparative advantage from

\footnotetext{
${ }^{12}$ If there are many equilibria, pick any one for the comparison to follow.
} 
FIGURE 2

EXPECTED COST AND CRITICAL COST REDUCTION $\widehat{\gamma}$

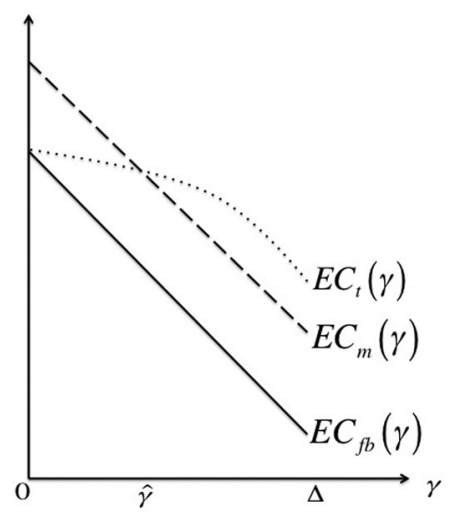

referrals is important, but that experts operate as solo-practitioners when work effort is more important. Using US census data, Garicano and Hubbard (2009) show that lawyers form multispecialty partnerships when they consult for corporations in markets such as banking, environment, and real estate developments. Our theory provides the foundation for the claim in Garicano and Hubbard (2009). The complexity in commercial dealing likely calls for disparate knowledge, so cross-field referrals are critical. Lawyers in domestic, insurance, and criminal litigations more likely work as independent practitioners. Noncommercial cases are more idiosyncratic, so a lawyer's own effort is more critical.

Another illustration of our theory is in the malpractice liability and personal litigations. According to Parikh (2006/2007), "top-end" lawyers in medical malpractice and product liability work in large practices, but "low-end" lawyers in automobile and "slip-and-fall" accidents work in solo practices. Our theory provides the rationale for the difference in the work organization of these lawyers. Top-end lawyers deal with more complex cases, so coordination between experts is important. By contrast, low-end lawyers may not have to rely on referrals that often.

Referral fees and fee-splitting are common among legal professionals, so our model applies straightforwardly. Nevertheless, our theory can also provide a normative view on the healthcare sector. In most countries, medical doctors are prohibited from obtaining financial benefits when they refer patients. The restriction is likely a safeguard against conflict of interests. ${ }^{13}$ In our model, referral is a financial transaction, so it is inconsistent with the current practice. However, the US healthcare reform encourages providers to form Accountable Care Organizations (ACOs), which group together multispecialty providers, hospitals, and ambulatory care services.

Within an ACO, information about patients, procedures, and costs is shared. On the one hand, coordination of care among specialists is particularly important for efficient care delivery. On the other hand, as Frandsen and Rebitzer (2015) point out, free-riding problems in ACOs can be severe. These two issues are illustrated by Proposition 5: cost comparative advantage must be balanced against muted work incentives in ACOs. Early evidence about the performance of ACOs is mixed. ${ }^{14}$ Colla et al. (2013) document reduced spending for high-risk Medicare patients such

${ }^{13}$ However, Pauly (1979) argues that referral with prices can improve patient welfare when markets are imperfectly competitive. Biglaiser and Ma (2007) show that a physician's self-referral at a price may lead to higher quality, thus benefitting some consumers.

${ }^{14}$ See Health Affairs blog, July 25, 2013, at healthaffairs.org/blog/2013/07/25/taking-stock-of-initial-year-oneresults-for-pioneer-acos. 
as cancer patients. Similarly, the Centers for Medicare and Medicaid Services find that pioneer ACOs benefit high-risk patients, many of whom have multiple chronic conditions. ${ }^{15}$

\section{Robustness}

- We now discuss a number of robustness issues with the basic model of market referral. First, we assume only two states, $\omega_{1}$ and $\omega_{2}$. This can be regarded as a normalization, given that we consider only two experts. If there are many (even a continuum of) states, then we proceed by first defining the subset of states for which Expert 1 is less expensive than Expert 2, and then call that subset $\omega_{1}$. Second, we assume that the two states are equally likely. If they are not, the posterior probabilities in (1) will be modified by prior probabilities attached to the conditional densities $f_{1}$ and $f_{2}$. However, MLRP is unaffected, and it remains valid that Expert 2's cost of providing service to a client is lower than Expert 1 if and only if the client's signal is higher than a threshold. Our computation is made easier by states being equally likely, but this assumption does not lead to any conceptual difficulties.

We have ignored capacities and variable returns. Here, there is another source of cost comparative advantage. The initial matching process may favor, say, Expert 1, who now has too many clients. Decreasing returns may lead him to refer some clients to Expert 2 even before he undertakes any effort (so has received no signal). It is a complication that may interfere with the construction of Expert 2's equilibrium belief about the referred clients' states. An analysis will have to start with the initial match between clients and experts. However, we feel that this is beyond the scope of our current research.

Capacity and variable returns may also change the comparison between market and organization. Clearly, an organization is better able to enjoy economies of scale, manage capacities, or both. The market is likely better modelled by a random initial match, but an organization can channel clients to its experts efficiently. The details in Proposition 5 may have to be altered, but the basic principle of trade-off between adverse selection and cost-reduction incentive remains valid.

Next, we discuss two issues in details. First, we endogenize the tariff $T$ rather than take it as given. Second, we study the equilibrium of the referral game when the cost advantage $\Delta$ is larger than the average cost, relaxing our assumption $\Delta<\left(c_{L}+c_{H}\right) / 2$.

Equilibrium tariff. We modify the referral market game in Section 3 to include a Bertrandcompetition game. That is, in Stage 0, at the time when the clients' types are drawn, each expert announces a tariff. Consumers observe these tariffs, and choose an expert for service. A consumer promises to pay the required tariff to the chosen expert or to a referred expert, if any, when service is provided..$^{16}$ Because we have assumed that each expert provides the same benefit to a client, a consumer's equilibrium strategy must be to pick the expert who offers the lower tariff. If both experts announce the same tariff, a consumer will be indifferent between choosing either of them, and we specify that all consumers choose Expert 1.

Recall that a client's ex ante expected service cost is $\left(c_{L}+c_{H}\right) / 2$. We now construct an equilibrium in which both experts set tariffs at $\left(c_{L}+c_{H}\right) / 2$. First, we specify that given any announced pair of tariffs and consumers' equilibrium strategy, the continuation is the equilibrium in Proposition 3 in Section 3.

Suppose that Expert 1 sets tariff $T=\left(c_{L}+c_{H}\right) / 2$. Expert 2 gets no client if he charges the same or more. Furthermore, according to the equilibrium in Proposition 3, Expert 1's referral

\footnotetext{
${ }^{15}$ For a survey of ACOs data released by the Centers for Medicare and Medicaid Services on Pioneer ACO Model participants, see Brookings blog, October 13, 2014, "A More Complete Picture of Pioneer ACO Results" at www.brookings.edu/blogs/up-front/posts/2014/10/09-pioneer-aco-results-mcclellan\#recent_rr/.

${ }^{16}$ An expert cannot revise the tariff after he has exerted effort and obtained the signal. Both effort and signal are unobserved to the consumer. The issue of commitment is beyond the scope here. Furthermore, an expert cannot dump a client after the signal has been observed.
} 
offer will give him a zero-expected payoff. Therefore, Expert 2's payoff is zero if he charges $T=\left(c_{L}+c_{H}\right) / 2$ or more. Next, if Expert 2 undercuts, he gets all clients. Again, according to Proposition 3 , he takes no effort and does not refer, so makes a strictly negative expected profit. We conclude that it is a best response for Expert 2 to set $T=\left(c_{L}+c_{H}\right) / 2$.

Suppose that Expert 2 sets tariff $T=\left(c_{L}+c_{H}\right) / 2$. Expert 1 gets no client if he charges more. Furthermore, according to Proposition 3, Expert 1 gets no referral from Expert 2, so his payoff is zero. Given that all consumers choose him if he sets his tariff at $\left(c_{L}+c_{H}\right) / 2$, Expert 1 has no reason to undercut. We conclude that it is a best response for Expert 1 to set $T=\left(c_{L}+c_{H}\right) / 2$. Finally, it is straightforward to show that there is no equilibrium in which experts offer tariffs strictly above $\left(c_{L}+c_{H}\right) / 2$. At any such tariffs, Expert 2 will undercut.

Our construction is similar to a standard Bertrand game with firms having different (and constant) marginal production costs: in equilibrium, the more efficient firm sets a price equal to the marginal cost of the less efficient firm. Expert 1 is more efficient because he invests in information acquisition in the continuation equilibrium. Here, the "more efficient" Expert 1 sets the same tariff as the "less efficient" Expert 2, but takes all the surplus from trade. In equilibrium, all clients initially seek services from Expert 1, who later refers some to Expert $2 .{ }^{17}$

Large cost comparative advantage. We have assumed that the cost comparative advantage parameter $\Delta$ is smaller than $\left(c_{H}-c_{L}\right) / 2$, so for both experts, the service cost in state $\omega_{1}$ is lower than in state $\omega_{2}$. This is our interpretation for the state $\omega_{1}$ being good and state $\omega_{2}$ being bad. However, the value of $\Delta$ can be larger than $\left(c_{H}-c_{L}\right) / 2$. In this case, we have $c_{H}-\Delta<c_{L}+\Delta$. For Expert 2, if the client's state is $\omega_{1}$, the service cost becomes higher than if the state is $\omega_{2}$. Now, to Expert 2, $\omega_{1}$ looks like a bad state, whereas $\omega_{2}$ looks like a good state (but the opposite is true for Expert 1). This cost specification actually allows equilibrium referrals from each expert to the other.

The derivation of Expert 1's equilibrium strategy, and Expert 2's beliefs remain unchanged, and Proposition 4 in Section 3 continues to hold. We only wish to note that Expert 2's expected cost of providing service is decreasing in Expert 1's referral threshold, so the expression in (10) is decreasing in $\widehat{s}$; in Figure 1, the dashed line is downward sloping.

For Expert 2, suppose now that he has taken effort $e_{2}$. We construct an equilibrium strategy for Expert 2's referral and effort. Again, in an equilibrium, Expert 1 accepts a referral if the price is below a threshold, say, $p_{2}$. Given the tariff, if Expert 2 uses effort $e_{2}$ and receives signal $s$, he refers if and only if

$$
p_{2} \geq T-\frac{\left(c_{L}+\Delta\right) f_{1}\left(s \mid e_{2}\right)}{f_{1}\left(s \mid e_{2}\right)+f_{2}\left(s \mid e_{2}\right)}-\frac{\left(c_{H}-\Delta\right) f_{2}\left(s \mid e_{2}\right)}{f_{1}\left(s \mid e_{2}\right)+f_{2}\left(s \mid e_{2}\right)} .
$$

By MLRP, and $c_{L}+\Delta>c_{H}-\Delta$, the expected cost in (24) is decreasing in $s$, so the right-hand side of (24) is increasing in $s$. By passive belief, in equilibrium, the signal threshold for Expert 2's referral at $p_{2}$ is $\widehat{s}_{2}$ such that (24) holds as an equality at $s=\widehat{s}_{2}$. In equilibrium, Expert 2 refers a client to Expert 1 if and only if $s<\widehat{s}_{2}$. This is the key difference. A higher value of the signal $s$ indicates a higher likelihood of state $\omega_{2}$. Expert 2's expected cost is decreasing in the signal, so he refers a client if and only if the signal is lower than a threshold. This is favorable news to Expert 1.

\footnotetext{
${ }^{17}$ Clients do know about experts' cost comparative advantage. (In equilibrium, they pick Expert 1 even when both offer the same tariff.) If they do not, their strategies must not depend on experts' identities, so clients pick experts randomly when tariffs are identical. An equilibrium may then fail to exist when tariffs can be chosen from a continuous set. The more efficient Expert 1 always undercuts slightly. The usual way to restore existence is to discretize possible tariff offers. If the difference between possible tariffs is sufficiently small (like one cent), Expert 1 will just undercut to capture all clients when Expert 2 offers $T=\left(c_{L}+c_{H}\right) / 2$.
} 
Expert 1 receives all those clients with signals below $\widehat{s}_{2}$, so he accepts Expert 2's referral if and only if

$$
T-\frac{c_{L} \int_{\underline{\underline{s}}}^{\widehat{s_{2}}} f_{1}\left(x \mid e_{2}\right) \mathrm{d} x+c_{H} \int_{\underline{s}}^{\widehat{s_{2}}} f_{2}\left(x \mid e_{2}\right) \mathrm{d} x}{\int_{\underline{s}}^{\widehat{s_{2}}} f_{1}\left(x \mid e_{2}\right) \mathrm{d} x+\int_{\underline{s}}^{\widehat{s_{2}}} f_{2}\left(x \mid e_{2}\right) \mathrm{d} x} \geq p_{2},
$$

where the various integrals average out those signals below $\widehat{s}_{2}$ across the two states. Given effort $e_{2}$, an equilibrium in referrals exists if there are price $p_{2}$ and threshold $\widehat{s}_{2}$ such that

$$
\begin{aligned}
& T-\frac{\left(c_{L}+\Delta\right) f_{1}\left(\widehat{S}_{2} \mid e_{2}\right)+\left(c_{H}-\Delta\right) f_{2}\left(\widehat{s}_{2} \mid e_{2}\right)}{f_{1}\left(\widehat{s}_{2} \mid e_{2}\right)+f_{2}\left(\widehat{s}_{2} \mid e_{2}\right)}=p_{2} \\
& =T-\frac{c_{L} \int_{\underline{s}}^{\widehat{s_{2}}} f_{1}\left(x \mid e_{2}\right) \mathrm{d} x+c_{H} \int_{\underline{s}}^{\widehat{s_{2}}} f_{2}\left(x \mid e_{2}\right) \mathrm{d} x}{\int_{\underline{s}}^{\widehat{s_{2}}} f_{1}\left(x \mid e_{2}\right) \mathrm{d} x+\int_{\underline{\underline{s}}}^{\widehat{s_{2}}} f_{2}\left(x \mid e_{2}\right) \mathrm{d} x} .
\end{aligned}
$$

This is the characterization of the referral equilibrium for Expert 2, as Proposition 1 is for Expert 1. Such price $p_{2}$ and threshold $\widehat{s}_{2}$ satisfying (25) must exist. Indeed, by MLRP, $c_{L}<c_{H}$, and $c_{L}+\Delta>c_{H}-\Delta$, the ratio in the left-hand side of (25) is decreasing in $\widehat{s}_{2}$, whereas the ratio in the right-hand side is increasing. ${ }^{18}$

For the continuation equilibrium with price $p_{2}$ and threshold $\widehat{s}_{2}$, Expert 2's per-client expected payoff from effort $e_{2}$ can be simplified to

$$
\begin{aligned}
& {\left[T-\frac{c_{L}+c_{H}}{2}\right]+0.5 \int_{\underline{s}}^{\widehat{s_{2}}}\left\{\left[p_{2}-\left(T-c_{L}-\Delta\right)\right] f_{1}\left(x \mid e_{2}\right)\right.} \\
& \left.\left.+\left[p_{2}-\left(T-c_{H}+\Delta\right)\right] f_{2}\left(x \mid e_{1}\right)\right]\right\} \mathrm{d} x-\phi\left(e_{2}\right) .
\end{aligned}
$$

This has the same interpretation of Expert 1's expected payoff in (13). Expert 2's optimal effort is one that maximizes (26), and its first-order condition is

$$
0.5 \int_{\underline{s}}^{\widehat{s}_{2}}\left\{\left[p_{2}-\left(T-c_{L}-\Delta\right)\right] \frac{\partial f_{1}\left(x \mid e_{2}\right)}{\partial e_{2}}+\left[p_{2}-\left(T-c_{H}+\Delta\right)\right] \frac{\partial f_{2}\left(x \mid e_{2}\right)}{\partial e_{2}}\right\} \mathrm{d} x=\phi^{\prime}\left(e_{2}\right) \text {. }
$$

Expert 2's equilibrium strategy is therefore characterized by price $p_{2}$, threshold $\widehat{s}_{2}$, and effort $e_{2}$ satisfying (25) and (27).

Using the same steps as in the proof of Proposition 4, we can show that Expert 2's equilibrium effort is never first best, and may be higher or lower. Furthermore, given the equilibrium effort $e_{2}$, Expert 2's equilibrium referral threshold $\widehat{s}_{2}$ satisfies $f_{2}\left(\widehat{s}_{2} \mid e_{2}\right)<f_{1}\left(\widehat{s}_{2} \mid e_{2}\right)$, so Expert 2 sometimes retains a client even when his expected service cost is higher than Expert 1's.

\section{Conclusion}

We posit a theory about how an organization can overcome market frictions due to hidden action and hidden information. This is a novel approach in the study of credence goods. The extant literature has looked at individual experts operating in a market to serve clients. There has been a lack of focus on how organizations may change experts' incentives. Although an organization can overcome adverse selection by the cost-transfer protocol, this leads to reduced work incentives. We derive a theory of the firm based on cost of adverse selection in the market compared to cost of reduced work incentive within an organization.

We have maintained some simplifications in our model. It may be interesting to study the referral game when clients' benefits, not just their costs, are uncertain. Can referral convey information about benefits? Can a client rely on an expert to tell him that a service is not

\footnotetext{
${ }^{18}$ MLRP implies that the distribution $f_{1}$ is first-order stochastically dominated by $f_{2}$.
} 
worthwhile? Our experts are profit maximizers. If one considers the health market as a specific application, physicians are known to be altruistic, so the pure profit-maximization assumption is invalid. It will be interesting to study how altruistic experts will play the referral game. However, in other organizations, collusion among experts may be expected. In the spirit of Gromb and Martimort (2007) who model how a principal may prevent or allow collusion, we can study trade-off between experts using information for efficiency and experts exploiting information for collusion.

In the details of our model, we have also made a number of assumptions. Multiple rounds of information efforts are assumed away. Nor are multiple rounds of referral price offers allowed. We have also made use of the constant returns to scale in services. Any of these issues may be relaxed for a more general model.

\section{Appendix}

This Appendix contains the proofs of Lemmas 3, 4, 5 and of Propositions 1, 2, 3, 4, 5.

Proof of Lemma 3. By MLRP, $\frac{f_{2}\left(x \mid e_{1}\right)}{f_{1}\left(x \mid e_{1}\right)}$ is increasing in $x$, so for any $s$, we have

$$
\begin{aligned}
\frac{\int_{s}^{\bar{s}} f_{2}\left(x \mid e_{1}\right) \mathrm{dx}}{\int_{s}^{\bar{s}} f_{1}\left(x \mid e_{1}\right) \mathrm{dx}} & \equiv \frac{\int_{s}^{\bar{s}} \frac{f_{2}\left(x \mid e_{1}\right)}{f_{1}\left(x \mid e_{1}\right)} \cdot f_{1}\left(x \mid e_{1}\right) \mathrm{dx}}{\int_{s}^{\bar{s}} f_{1}\left(x \mid e_{1}\right) \mathrm{dx}} \\
& >\frac{\int_{s}^{\bar{s}} \frac{f_{2}\left(s \mid e_{1}\right)}{f_{1}\left(s \mid e_{e}\right)} f_{1}\left(x \mid e_{1}\right) \mathrm{dx}}{\int_{s}^{\bar{s}} f_{1}\left(x \mid e_{1}\right) \mathrm{dx}}=\frac{f_{2}\left(s \mid e_{1}\right)}{f_{1}\left(s \mid e_{1}\right)}
\end{aligned}
$$

It follows that

$$
\frac{\int_{s}^{\bar{s}} f_{1}\left(x \mid e_{1}\right) \mathrm{dx}}{\int_{s}^{\bar{s}} f_{1}\left(x \mid e_{1}\right) \mathrm{dx}+\int_{\mathrm{s}}^{\bar{s}} \mathrm{f}_{2}\left(\mathrm{x} \mid \mathrm{e}_{1}\right) \mathrm{dx}}<\frac{f_{1}\left(s \mid e_{1}\right)}{f_{1}\left(s \mid e_{1}\right)+f_{2}\left(s \mid e_{1}\right)}
$$

and

$$
\frac{\int_{s}^{s} f_{2}\left(x \mid e_{1}\right) \mathrm{d} x}{\int_{s}^{s} f_{1}\left(x \mid e_{1}\right) \mathrm{d} x+\int_{s}^{s} f_{2}\left(x \mid e_{1}\right) \mathrm{d} x}>\frac{f_{2}\left(s \mid e_{1}\right)}{f_{1}\left(s \mid e_{1}\right)+f_{2}\left(s \mid e_{1}\right)} .
$$

Therefore, at any $s<\bar{s}$,

$$
\frac{c_{L} \int_{s}^{\bar{s}} f_{1}\left(x \mid e_{1}\right) \mathrm{d} x+c_{H} \int_{s}^{\bar{s}} f_{2}\left(x \mid e_{1}\right) \mathrm{d} x}{\int_{s}^{\bar{s}} f_{1}\left(x \mid e_{1}\right) \mathrm{d} x+\int_{s}^{\bar{s}} f_{2}\left(x \mid e_{1}\right) \mathrm{d} x}>\frac{c_{L} f_{1}\left(s \mid e_{1}\right)+c_{H} f_{2}\left(s \mid e_{1}\right)}{f_{1}\left(s \mid e_{1}\right)+f_{2}\left(s \mid e_{1}\right)} .
$$

Applying L'Hospital's rule, we have

$$
\begin{array}{r}
\lim _{s \rightarrow \bar{s}} \frac{\left(c_{L}+\Delta\right) \int_{s}^{\bar{s}} f_{1}\left(x \mid e_{1}\right) \mathrm{d} x+\left(c_{H}-\Delta\right) \int_{s}^{\bar{s}} f_{2}\left(x \mid e_{1}\right) \mathrm{d} x}{\int_{s}^{\bar{s}} f_{1}\left(x \mid e_{1}\right) \mathrm{d} x+\int_{s}^{\bar{s}} f_{2}\left(x \mid e_{1}\right) \mathrm{d} x}=\frac{\left(c_{L}+\Delta\right) f_{1}\left(\bar{s} \mid e_{1}\right)+\left(c_{H}-\Delta\right) f_{2}\left(\bar{s} \mid e_{1}\right)}{f_{1}\left(\bar{s} \mid e_{1}\right)+f_{2}\left(\bar{s} \mid e_{1}\right)} \\
=\frac{c_{L} f_{1}\left(\bar{s} \mid e_{1}\right)+c_{H} f_{2}\left(\bar{s} \mid e_{1}\right)-\Delta\left[f_{2}\left(\bar{s} \mid e_{1}\right)-f_{1}\left(\bar{s} \mid e_{1}\right)\right]}{f_{1}\left(\bar{s} \mid e_{1}\right)+f_{2}\left(\bar{s} \mid e_{1}\right)}<\frac{c_{L} f_{1}\left(\bar{s} \mid e_{1}\right)+c_{H} f_{2}\left(\bar{s} \mid e_{1}\right)}{f_{1}\left(\bar{s} \mid e_{1}\right)+f_{2}\left(\bar{s} \mid e_{1}\right)} .
\end{array}
$$

We have shown that at $s$ sufficiently near $\bar{s}$, (10) is smaller than (9).

Now at $\underline{s}$, we have

$$
\begin{aligned}
\frac{\left(c_{L}+\Delta\right) \int_{\underline{s}}^{\bar{s}} f_{1}\left(x \mid e_{1}\right) \mathrm{d} x+\left(c_{H}-\Delta\right) \int_{\underline{\underline{s}}}^{\bar{s}} f_{2}\left(x \mid e_{1}\right) \mathrm{d} x}{\int_{\underline{s}}^{\bar{s}} f_{1}\left(x \mid e_{1}\right) \mathrm{d} x+\int_{\underline{\underline{s}}}^{\bar{s}} f_{2}\left(x \mid e_{1}\right) \mathrm{d} x} & =\frac{c_{L} \int_{\underline{s}}^{\bar{s}} f_{1}\left(x \mid e_{1}\right) \mathrm{d} x+c_{H} \int_{\underline{s}}^{\bar{s}} f_{2}\left(x \mid e_{1}\right) \mathrm{d} x}{\int_{\underline{s}}^{\bar{s}} f_{1}\left(x \mid e_{1}\right) \mathrm{d} x+\int_{\underline{s}}^{\bar{s}} f_{2}\left(x \mid e_{1}\right) \mathrm{d} x} \\
& >\frac{c_{L} f_{1}\left(\underline{s} \mid e_{1}\right)+c_{H} f_{2}\left(\underline{s} \mid e_{1}\right)}{f_{1}\left(\underline{s} \mid e_{1}\right)+f_{2}\left(\underline{e_{1}} \mid e_{1}\right)} .
\end{aligned}
$$

We have shown that at $s$ sufficiently near $\underline{s},(10)$ is larger than (9). Therefore, the equation in the lemma must have a solution $\widehat{s}$.

Finally, for uniqueness, rewrite the equation in the lemma as

$$
\frac{\left(c_{L}+\Delta\right)+\left(c_{H}-\Delta\right) \frac{\left[\int_{s}^{s} f_{2}\left(x \mid e_{1}\right) \mathrm{d} x\right] / f_{2}\left(s \mid e_{1}\right)}{\left.\left[\int_{s}^{s} f_{1}\left(x \mid e_{1}\right) \mathrm{d} x\right] / f_{1} s \mid e_{1}\right)} \times \frac{f_{2}\left(s \mid e_{1}\right)}{f_{1}\left(s \mid e_{1}\right)}}{1+\frac{\left[\int_{s}^{s} f_{2}\left(x \mid e_{1}\right) \mathrm{d} x\right] / f_{2}\left(s \mid e_{1}\right)}{\left.\left[\int_{s}^{s} f_{1}\left(x \mid e_{1}\right) \mathrm{d} x\right] / f_{1} s \mid e_{1}\right)} \times \frac{f_{2}\left(s \mid e_{1}\right)}{f_{1}\left(s \mid e_{1}\right)}}=\frac{c_{L}+c_{H} \frac{f_{2}\left(s \mid e_{1}\right)}{f_{1}\left(s \mid e_{1}\right)}}{1+\frac{f_{2}\left(s \mid e_{1}\right)}{f_{1}\left(s \mid e_{1}\right)}} .
$$


By MLRP, the inverse hazard rates satisfy $\left[\int_{s}^{\bar{s}} f_{2}\left(x \mid e_{1}\right) \mathrm{d} x\right] / f_{2}\left(s \mid e_{1}\right)>\left[\int_{s}^{\bar{s}} f_{1}\left(x \mid e_{1}\right) \mathrm{d} x\right] / f_{1}\left(s \mid e_{1}\right)$; see also (A1) above. As $s$ changes, the rates of change of the left-hand and right-hand sides of (A3) will never be identical. As separate functions, the graphs of (9) and (10) can only cross each other once. In other words, there can only be one solution.

Proof of Proposition 1. The two equations in (12) include the equation in Lemma 3, which already establishes a solution for $\widehat{s}$. We then set the value of $p_{1}$ according to (12). From Lemma 1, equilibrium referrals are those with signals above a threshold, so we simply set Expert 1's referral threshold at $\widehat{s}$. From Lemma 1, Expert 2 accepts a referral if and only if the price is below a threshold, so we set Expert 2's acceptance threshold at $p_{1}$.

Proof of Proposition 2. Assume, to the contrary, that Expert 2 exerts a strictly positive effort $e_{2}$ in an equilibrium. By Lemma 1, Expert 2 refers a client if and only if the client's signal is above a threshold, say, $\widetilde{s}$. Let this referral be made at a price $p_{2}$, which will be accepted by Expert 1 in equilibrium.

At signal $\widetilde{s}$, Expert 2's expected cost is $\left(c_{L}+\Delta\right) \operatorname{Pr}\left(\omega_{1} \mid \widetilde{s}, e_{2}\right)+\left(c_{H}-\Delta\right) \operatorname{Pr}\left(\omega_{2} \mid \widetilde{s}, e_{2}\right)$

$$
\begin{gathered}
=\frac{\left(c_{L}+\Delta\right) f_{1}\left(\widetilde{s} \mid e_{2}\right)+\left(c_{H}-\Delta\right) f_{2}\left(\widetilde{s} \mid e_{2}\right)}{f_{1}\left(\widetilde{s} \mid e_{2}\right)+f_{2}\left(\widetilde{s} \mid e_{2}\right)} \\
<\frac{\left(c_{L}+\Delta\right) \int_{\widetilde{s}}^{\bar{s}} f_{1}\left(x \mid e_{2}\right) \mathrm{d} x+\left(c_{H}-\Delta\right) \int_{\widetilde{s}}^{\bar{s}} f_{2}\left(x \mid e_{2}\right) \mathrm{d} x}{\int_{\widetilde{s}}^{\bar{s}} f_{1}\left(x \mid e_{2}\right) \mathrm{d} x+\int_{\widetilde{s}}^{\bar{s}} f_{2}\left(x \mid e_{2}\right) \mathrm{d} x} \\
<\frac{c_{L} \int_{\widetilde{s}}^{\bar{s}} f_{1}\left(x \mid e_{2}\right) \mathrm{d} x+c_{H} \int_{\widetilde{s}}^{\bar{s}} f_{2}\left(x \mid e_{2}\right) \mathrm{d} x}{\int_{\widetilde{s}}^{\bar{s}} f_{1}\left(x \mid e_{2}\right) \mathrm{d} x+\int_{\widetilde{s}}^{\bar{s}} f_{2}\left(x \mid e_{2}\right) \mathrm{d} x},
\end{gathered}
$$

where the inequality in (A4) follows from MLRP (see also (A2) in the proof of Lemma 3). Now the derivative of (A4) with respect to $\Delta$ is

$$
\frac{\int_{\tilde{s}}^{\bar{s}} f_{1}\left(x \mid e_{2}\right) \mathrm{d} x-\int_{\tilde{s}}^{\bar{s}} f_{2}\left(x \mid e_{2}\right) \mathrm{d} x}{\int_{\tilde{s}}^{\bar{s}} f_{1}\left(x \mid e_{2}\right) \mathrm{d} x+\int_{\tilde{s}}^{\bar{s}} f_{2}\left(x \mid e_{2}\right) \mathrm{d} x}<0,
$$

where the inequality is due to $f_{2}\left(\cdot \mid e_{2}\right)$ first-order stochastically dominating $f_{1}\left(\cdot \mid e_{2}\right)$, an implication of MLRP. Hence, (A4) is decreasing in $\Delta$. By reducing the value of $\Delta$ to zero, we obtain (A5), Expert 1's expected cost of providing service to a client conditional on Expert 2's signal being at least $\widetilde{s}$.

In sum, because

$$
\frac{\left(c_{L}+\Delta\right) f_{1}\left(\widetilde{s} \mid e_{2}\right)+\left(c_{H}-\Delta\right) f_{2}\left(\widetilde{s} \mid e_{2}\right)}{f_{1}\left(\widetilde{s} \mid e_{2}\right)+f_{2}\left(\widetilde{s} \mid e_{2}\right)}<\frac{c_{L} \int_{\widetilde{s}}^{\bar{s}} f_{1}\left(x \mid e_{2}\right) \mathrm{d} x+c_{H} \int_{\widetilde{s}}^{\bar{s}} f_{2}\left(x \mid e_{2}\right) \mathrm{d} x}{\int_{\widetilde{s}}^{\bar{s}} f_{1}\left(x \mid e_{2}\right) \mathrm{d} x+\int_{\widetilde{s}}^{\bar{s}} f_{2}\left(x \mid e_{2}\right) \mathrm{d} x},
$$

it is impossible to find $p_{2}$ to satisfy

$$
T-\frac{\left(c_{L}+\Delta\right) f_{1}\left(\widetilde{s} \mid e_{2}\right)+\left(c_{H}-\Delta\right) f_{2}\left(\widetilde{s} \mid e_{2}\right)}{f_{1}\left(\widetilde{s} \mid e_{2}\right)+f_{2}\left(\widetilde{s} \mid e_{2}\right)} \leq p_{2} \leq T-\frac{c_{L} \int_{\widetilde{s}}^{\bar{s}} f_{1}\left(x \mid e_{2}\right) \mathrm{d} x+c_{H} \int_{\widetilde{s}}^{\bar{s}} f_{2}\left(x \mid e_{2}\right) \mathrm{d} x}{\int_{\widetilde{s}}^{\bar{s}} f_{1}\left(x \mid e_{2}\right) \mathrm{d} x+\int_{\widetilde{s}}^{\bar{s}} f_{2}\left(x \mid e_{2}\right) \mathrm{d} x},
$$

a condition for an equilibrium. This is a contradiction.

Proof of Proposition 3. First, $\left(e_{1}^{*}, \widehat{s}^{*}\right)$ maximize Expert 1's expected utility (13) given Expert 2's acceptance threshold $p_{1}^{*}$, so this is a best response. Second, $p_{1}^{*}$ is given by (12) at $\widehat{s}$ and $e_{1}^{*}$, so acceptance threshold $p_{1}^{*}$ is a best response. Expert 2 's belief clearly satisfies passive belief.

We now show that Expert 2's best response is to choose no effort. Given the most pessimistic belief, Expert 1 will reject any referral price $p$ where $T-\operatorname{Pr}\left(\omega_{1} \mid \bar{s}, \widetilde{e}\right) c_{L}-\operatorname{Pr}\left(\omega_{2} \mid \bar{s}, \widetilde{e}\right) c_{H}<p$, so the minimum price for Expert 1 to accept a referral is $p=T-\operatorname{Pr}\left(\omega_{1} \mid \bar{s}, \widetilde{e}\right) c_{L}-\operatorname{Pr}\left(\omega_{2} \mid \bar{s}, \widetilde{e}\right) c_{H}$.

Suppose that Expert 2 takes some effort, say, $e_{2}>0$. Expert 2's expected utility from keeping the client at signal $s$ is $T-\operatorname{Pr}\left(\omega_{1} \mid s, e_{2}\right)\left(c_{L}+\Delta\right)-\operatorname{Pr}\left(\omega_{2} \mid s, e_{2}\right)\left(c_{H}+\Delta\right)$. By definition, $\operatorname{Pr}\left(\omega_{2} \mid s, e_{2}\right) \leq \operatorname{Pr}\left(\omega_{2} \mid \bar{s}, \widetilde{e}\right)$, so we have

$$
\begin{aligned}
\operatorname{Pr}\left(\omega_{1} \mid s, e_{2}\right)\left(c_{L}+\Delta\right)+\operatorname{Pr}\left(\omega_{2} \mid s, e_{2}\right)\left(c_{H}-\Delta\right) & \leq \operatorname{Pr}\left(\omega_{1} \mid \bar{s}, \widetilde{e}\right)\left(c_{L}+\Delta\right)+\operatorname{Pr}\left(\omega_{2} \mid \bar{s}, \widetilde{e}\right)\left(c_{H}-\Delta\right) \\
& <\operatorname{Pr}\left(\omega_{1} \mid \bar{s}, \widetilde{e}\right) c_{L}+\operatorname{Pr}\left(\omega_{2} \mid \bar{s}, \widetilde{e}\right) c_{H} .
\end{aligned}
$$

Therefore,

$$
T-\operatorname{Pr}\left(\omega_{1} \mid s, e_{2}\right)\left(c_{L}+\Delta\right)-\operatorname{Pr}\left(\omega_{2} \mid s, e_{2}\right)\left(c_{H}-\Delta\right)>T-\operatorname{Pr}\left(\omega_{1} \mid \bar{s}, \widetilde{e}\right) c_{L}-\operatorname{Pr}\left(\omega_{2} \mid \bar{s}, \widetilde{e}\right) c_{H}=p .
$$

Expert 2 cannot profit from deviating to an effort and referring some clients to Expert 1.

It remains to show that the triple $\left[e_{1}^{*}, \widehat{s}^{*}, p_{1}^{*}\right]$ exists. We use a standard fixed-point argument. Bound Expert 1's feasible efforts by a compact convex set, say, a closed interval of the real numbers. Clearly we can let the referral threshold reside in the signal support, which is convex and compact. Finally, we can also let the referral price be an element of a 
compact convex set of real numbers. Define a map $\Psi$ that takes an effort, a referral threshold, and a price onto themselves: $\Psi\left(e_{1}, \widehat{s}, p_{1}\right)=\left(e_{1}^{\prime}, \widehat{s}^{\prime}, p_{1}^{\prime}\right)$, where we define $\Psi$ by

$$
\begin{gathered}
\left.\left(e_{1}^{\prime}, \widehat{s}^{\prime}\right)=\operatorname{argmax}_{e_{1}, \widehat{s}} 0.5 \int_{\widehat{s}}^{\bar{s}}\left\{\left[p_{1}-\left(T-c_{L}\right)\right] f_{1}\left(x \mid e_{1}\right)+\left[p_{1}-\left(T-c_{H}\right)\right] f_{2}\left(x \mid e_{1}\right)\right]\right\} \mathrm{d} x-\phi\left(e_{1}\right) \\
p_{1}^{\prime}=T-\frac{\left(c_{L}+\Delta\right) \int_{\widehat{s}}^{\bar{s}} f_{1}\left(x \mid e_{1}\right) \mathrm{d} x+\left(c_{H}-\Delta\right) \int_{\widehat{s}}^{\bar{s}} f_{2}\left(x \mid e_{1}\right) \mathrm{d} x}{\int_{\widehat{s}}^{\bar{s}} f_{1}\left(x \mid e_{1}\right) \mathrm{d} x+\int_{\widehat{s}}^{\bar{s}} f_{2}\left(x \mid e_{1}\right) \mathrm{d} x} .
\end{gathered}
$$

Here, (A7) is Expert 1's best response against Expert 2's referral-acceptance price $p_{1}$ (the same as the maximization of (13) with respect to effort and referral threshold), whereas (A8) is Expert 2's referral-acceptance best response against Expert 1's effort $e_{1}$ and referral threshold $\widehat{s}$ (see also (12) in Proposition 1).

Clearly, the Maximum Theorem applies to (A7), and there is a selection of the solution $\left(e_{1}^{\prime}, \widehat{s}^{\prime}\right)$, which is continuous in $p_{1}$. Furthermore, $p_{1}^{\prime}$ in (A8) is obviously continuous in $e_{1}$ and $\widehat{s}$. By Brouwer's Fixed-Point Theorem, $\Psi$ has a fixed point $\left(e_{1}^{*}, \widehat{s}^{*}, p_{1}^{*}\right)$.

Proof of Proposition 4. Suppose not, that is, suppose that in an equilibrium, Expert 1's effort and referral threshold are first best. Then, $f_{2}\left(\widehat{s}^{*} \mid e_{1}^{*}\right)=f_{1}\left(\widehat{s}^{*} \mid e_{1}^{*}\right)$; see Section 2. From the second equation in (15), we obtain

$$
\begin{aligned}
& p_{1}^{*}-\left(T-c_{L}\right)=-\frac{\left(c_{H}-c_{L}\right) f_{2}\left(\widehat{s}^{*} \mid e_{1}^{*}\right)}{f_{1}\left(\widehat{s}^{*} \mid e_{1}^{*}\right)+f_{2}\left(\widehat{s}^{*} \mid e_{1}^{*}\right)}=-\frac{c_{H}-c_{L}}{2} \\
& p_{1}^{*}-\left(T-c_{H}\right)=\frac{\left(c_{H}-c_{L}\right) f_{1}\left(\widehat{s}^{*} \mid e_{1}^{*}\right)}{f_{1}\left(\widehat{s}^{*} \mid e_{1}^{*}\right)+f_{2}\left(\widehat{s}^{*} \mid e_{1}^{*}\right)}=\frac{c_{H}-c_{L}}{2} .
\end{aligned}
$$

We then write (16) as

$$
0.5\left[\frac{c_{H}-c_{L}}{2}\right] \int_{\widehat{s}^{*}}^{\bar{s}}\left\{\frac{\partial f_{2}\left(x \mid e_{1}^{*}\right)}{\partial e_{1}}-\frac{\partial f_{1}\left(x \mid e_{1}^{*}\right)}{\partial e_{1}}\right\} d x=\phi^{\prime}\left(e_{1}^{*}\right) .
$$

However, by assumption, $c_{H}-c_{L}>2 \Delta$. Comparing this simplified (16) with (5), we conclude that $e_{1}^{*}>e^{f b}$, so Expert 1 's effort is not first best.

Next, suppose, to the contrary, that $f_{2}\left(\widehat{s}^{*} \mid e_{1}^{*}\right) \leq f_{1}\left(\widehat{s}^{*} \mid e_{1}^{*}\right)$. First, we note that

$$
\frac{\left(c_{L}+\Delta\right) f_{1}\left(\widehat{s}^{*} \mid e_{1}^{*}\right)+\left(c_{H}-\Delta\right) f_{2}\left(\widehat{s}^{*} \mid e_{1}^{*}\right)}{f_{1}\left(\widehat{s}^{*} \mid e_{1}^{*}\right)+f_{2}\left(\widehat{s}^{*} \mid e_{1}^{*}\right)} \equiv \frac{c_{L} f_{1}\left(\widehat{s}^{*} \mid e_{1}^{*}\right)+c_{H} f_{2}\left(\widehat{s}^{*} \mid e_{1}^{*}\right)}{f_{1}\left(\widehat{s}^{*} \mid e_{1}^{*}\right)+f_{2}\left(\widehat{s}^{*} \mid e_{1}^{*}\right)}+\frac{\Delta\left[f_{1}\left(\widehat{s}^{*} \mid e_{1}^{*}\right)-f_{2}\left(\widehat{s}^{*} \mid e_{1}^{*}\right)\right]}{f_{1}\left(\widehat{s}^{*} \mid e_{1}^{*}\right)+f_{2}\left(\widehat{s}^{*} \mid e_{1}^{*}\right)} .
$$

Therefore, by $f_{2}\left(\widehat{s}^{*} \mid e_{1}^{*}\right) \leq f_{1}\left(\widehat{s}^{*} \mid e_{1}^{*}\right)$, we have

$$
\frac{c_{L} f_{1}\left(\widehat{s}^{*} \mid e_{1}^{*}\right)+c_{H} f_{2}\left(\widehat{s}^{*} \mid e_{1}^{*}\right)}{f_{1}\left(\widehat{s}^{*} \mid e_{1}^{*}\right)+f_{2}\left(\widehat{s}^{*} \mid e_{1}^{*}\right)} \leq \frac{\left(c_{L}+\Delta\right) f_{1}\left(\widehat{s}^{*} \mid e_{1}^{*}\right)+\left(c_{H}-\Delta\right) f_{2}\left(\widehat{s}^{*} \mid e_{1}^{*}\right)}{f_{1}\left(\widehat{s}^{*} \mid e_{1}^{*}\right)+f_{2}\left(\widehat{s}^{*} \mid e_{1}^{*}\right)}
$$

Now by MLRP, we have (A1):

$$
\frac{\int_{\widehat{s}^{*}}^{\bar{s}} f_{2}\left(x \mid e_{1}^{*}\right) \mathrm{d} x}{\int_{\widehat{s}^{*}}^{\bar{s}} f_{1}\left(x \mid e_{1}^{*}\right) \mathrm{d} x}>\frac{f_{2}\left(\widehat{s}^{*} \mid e_{1}^{*}\right)}{f_{1}\left(\widehat{s}^{*} \mid e_{1}^{*}\right)}
$$

It follows that

$$
\begin{aligned}
& \frac{\left(c_{L}+\Delta\right) \int_{\widehat{s}^{*}}^{\bar{s}} f_{1}\left(x \mid e_{1}^{*}\right) \mathrm{d} x+\left(c_{H}-\Delta\right) \int_{\widehat{s}^{*}}^{\bar{s}} f_{2}\left(x \mid e_{1}^{*}\right) \mathrm{d} x}{\int_{\widehat{s}^{*}}^{\bar{s}} f_{1}\left(x \mid e_{1}^{*}\right) \mathrm{d} x+\int_{\widehat{s}^{*}}^{\bar{s}} f_{2}\left(x \mid e_{1}^{*}\right) \mathrm{d} x} \\
> & \frac{\left(c_{L}+\Delta\right) f_{1}\left(\widehat{s}^{*} \mid e_{1}^{*}\right)+\left(c_{H}-\Delta\right) f_{2}\left(\widehat{s}^{*} \mid e_{1}^{*}\right)}{f_{1}\left(\widehat{s}^{*} \mid e_{1}^{*}\right)+f_{2}\left(\widehat{s}^{*} \mid e_{1}^{*}\right)} \\
\geq & \frac{c_{L} f_{1}\left(\widehat{s}^{*} \mid e_{1}^{*}\right)+c_{H} f_{2}\left(\widehat{s}^{*} \mid e_{1}^{*}\right)}{f_{1}\left(\widehat{s}^{*} \mid e_{1}^{*}\right)+f_{2}\left(\widehat{s}^{*} \mid e_{1}^{*}\right)},
\end{aligned}
$$

which contradicts (15). We conclude that $f_{2}\left(\widehat{s}^{*} \mid e_{1}^{*}\right)>f_{1}\left(\widehat{s}^{*} \mid e_{1}^{*}\right)$.

Proof of Lemma 4. The expression in the lemma is obtained from solving for the $f_{1} / f_{2}$ ratio in (18). Clearly, at $\gamma=0$, we have $f_{1}\left(\widetilde{s}_{1} \mid e_{1}\right)=f_{2}\left(\widetilde{s}_{1} \mid e_{1}\right)$, so $\widetilde{s}_{1}$ is the first-best referral threshold at effort $e_{1}$ : see $f_{1}\left(\widehat{s}^{f b} \mid e\right)=f_{2}\left(\widehat{s}^{f b} \mid e\right)$ in Section 2 . The right-hand side of (19) is strictly decreasing in $\gamma$, and goes to 0 as $\gamma$ increases to $\Delta$. By MLRP, we conclude that $\widetilde{s}_{1}$ must increase to $\bar{s}$.

Proof of Lemma 5. We drop all constants (those that involve only $c_{L}$ and $c_{H}$ ) in (20) and then simplify it to obtain

$$
-\int_{\underline{s}}^{\widetilde{s}_{1}} 0.5 \gamma\left[f_{1}\left(x \mid e_{1}\right)+f_{2}\left(x \mid e_{1}\right)\right] \mathrm{d} x-\int_{\widetilde{s}_{1}}^{\bar{s}} 0.5 \Delta\left[f_{2}\left(x \mid e_{1}\right)-f_{1}\left(x \mid e_{1}\right)\right] \mathrm{d} x+\phi\left(e_{1}\right) .
$$


Differentiating this with respect to $e_{1}$ and setting it to zero, we get the first-order condition:

$$
0.5\left\{\int_{\underline{s}}^{\widetilde{s}_{1}} \gamma\left[\frac{\partial f_{1}\left(x \mid e_{1}\right)}{\partial e_{1}}+\frac{\partial f_{2}\left(x \mid e_{1}\right)}{\partial e_{1}}\right] \mathrm{d} x+\int_{\widetilde{s}_{1}}^{\bar{s}} \Delta\left[\frac{\partial f_{2}\left(x \mid e_{1}\right)}{\partial e_{1}}-\frac{\partial f_{1}\left(x \mid e_{1}\right)}{\partial e_{1}}\right] \mathrm{d} x\right\}=\phi^{\prime}\left(e_{1}\right) .
$$

Now the first-best information effort is given by (5), and we conclude that $e_{1}$ is never first best, except at $\gamma=0$.

By Lemma 4, $\widetilde{s}_{1}$ tends to $\bar{s}$ as $\gamma$ tends to $\Delta$. The first integral in (A9) becomes arbitrarily small because the integrands are derivatives of densities, which sum to 0 over the support. Obviously, the second integral tends to 0 . Hence, any $e_{1}$ satisfying (A9) must tend to 0 .

Proof of Proposition 5. First, at $\gamma=0, E C_{t}(\gamma)$ in (4) is the expected cost at the first best (5). Also, at $\gamma=\Delta, \widetilde{s}_{1}=\bar{s}$, $\widetilde{s}_{2}=\underline{s}$, so $E C_{t}(\gamma)$ in (22) equals $\left\{\frac{c_{L}+c_{H}}{2}\right\}-\gamma$. Because $E C_{m}(\gamma)$ is the market equilibrium expected cost, it is higher than the first best. Hence, $E C_{m}(0)>E C_{t}(0)$. By inspection, we have $E C_{m}(\Delta)<E C_{t}(\Delta)$.

Next, because the market equilibrium is independent of $\gamma, E C_{m}(\gamma)$ has a derivative of -1 . The expected cost in $E C_{t}(\gamma)$ is the result of optimal choices of information effort and referral threshold, so the Envelope Theorem applies. The derivative of $E C_{t}(\gamma)$ is the partial derivative of (22) with respect to $\gamma$ :

$$
\frac{\mathrm{d} E C_{t}(\gamma)}{\mathrm{d} \gamma}=-0.5\left\{\int_{\underline{s}}^{\widetilde{s}_{1}}\left[f_{1}\left(x \mid \widetilde{e}_{1}\right)+f_{2}\left(x \mid \widetilde{e}_{1}\right)\right] \mathrm{d} x+\int_{\widetilde{s}_{2}}^{\bar{s}}\left[f_{1}\left(x \mid \widetilde{e}_{2}\right)+f_{2}\left(x \mid \widetilde{e}_{2}\right)\right] \mathrm{d} x\right\}>-1,
$$

where the inequality follows from $\widetilde{s}_{1}<\bar{s}$ and $\widetilde{s}_{2}>\underline{s}$. Hence, as $\gamma$ varies between 0 and $\Delta$, there is only point $\widehat{\gamma}$ such that $E C_{m}(\widehat{\gamma})=E C_{t}(\widehat{\gamma})$. The proposition follows.

\section{References}

ABLE, B.H. "The Stark Physician Self-Referral Law and Accountable Care Organizations: Collision Course or Opportunity to Reconcile Federal Anti-Abuse and Cost-Saving Legislation?" Journal of Law and Health, Vol. 26 (2013), pp 315-347.

ARbatskaya, M. AND Konishi, H. "Referrals in Search Markets.” International Journal of Industrial Organization, Vol. 30 (2012), pp. 89-101.

Bar-Isaac, H., Caruana, G., And Cunat, V., "Search Design and Market Structure." The American Economic Review, Vol. 102 (2012), pp. 1140-1160.

Biglaiser, G. And MA, C-T.A. "Moonlighting: Public Service and Private Practice.” RAND Journal of Economics, Vol. 38 (2007), pp. 1113-1133.

Bolton, P., Freixas, X., And Shapiro, J., "Conflicts of Interest, Information Provision, and Competition in the Financial Services Industry.” Journal of Financial Economics, Vol. 85 (2007), pp. 297-330.

Buehler, B. AND Schuett, F. "Certification and Minimum Quality Standards When Some Consumers are Uninformed." European Economic Review, Vol. 70 (2014), pp. 493-511.

Cebul, R.D., Rebitzer, J.B., Taylor, L.J., and Votruba, M.E. "Organizational Fragmentation and Care Quality in the U.S. Healthcare System.” Journal of Economic Perspectives, Vol. 22 (2008), pp. 93-113.

Colla, C.H., Lewis, V.A., Gottlieb, D.J., And Fisher, E.S. "Cancer Spending and Accountable Care Organizations: Evidence from the Physician Group Practice Demonstration.” Healthcare, Vol. 1 (2013), pp. 100-107.

DE FontenaY, C.C. And Gans, J.S. "Vertical Integration in the Presence of Upstream Competition." RAND Journal of Economics, Vol. 36 (2005), pp. 544-572.

Dequiedt, V. And Martimort, D. "Vertical Contracting with Informational Opportunism." American Economic Review, Vol. 105 (2015), pp. 2141-2182.

Dulleck, U. and KerschBamer, R. "On Doctors, Mechanics, and Computer Specialists: The Economics of Credence Goods.” Journal of Economic Literature, Vol. 44 (2006), pp. 5-42.

Epstein, A.J., Ketcham, J.D., And Nicholson, S. "Specialization and Matching in Professional Services Firms." RAND Journal of Economics, Vol. 41 (2010), pp. 811-834.

FrandSEn, B. AND ReBitZer, J.B. "Structuring Incentives within Organizations: The Case of Accountable Care Organizations." Journal of Law, Economics, \& Organization, Vol. 31 (2015), pp. 77-103.

Fuchs, W. And Garicano, L. "Matching Problems with Expertise in Firms and Markets." Journal of the European Economic Association, Vol. 8 (2010), pp. 354-364.

GARICANO, L. "Hierarchies and the Organization of Knowledge in Production." Journal of Political Economy, Vol. 108 (2000), pp. 874-904.

Garicano, L. AND HubBard, T.N. "Specialization, Firms, and Markets: The Division of Labor within and between Law Firms.” Journal of Law, Economics, \& Organization, Vol. 25 (2009), pp. 339-371.

Garicano, L. And Santos, T. "Referrals.” American Economic Review, Vol. 94 (2004), pp. 149-173.

Gromb, D. And Martimort, D. "Collusion and the Organization of Delegated Expertise." Journal of Economic Theory, Vol. 137 (2007), pp. 271-299.

Hart, O. AND Tirole, J. "Vertical Integration and Market Foreclosure.” Brookings Papers on Economic Activity, (1990), pp. 205-285. 
Inderst, R. And Ottaviani, M. "Misselling through Agents.” The American Economic Review, Vol. 99 (2009), pp. 883-908.

. "Competition through Commissions and Kickbacks.” American Economic Review, Vol. 102 (2012), pp. 780-809.

LAfFont, J.-J. AND Martimort, D. “Mechanism Design with Collusion and Correlation.” Econometrica, Vol. 68 (2000), pp. 309-342.

McAfee, P. And Schwartz, M. "Opportunism in Multilateral Vertical Contracting: Nondiscrimination, Exclusivity, and Uniformity.” American Economic Review, Vol. 84 (1994), pp. 210-230.

Mariñoso, B.G. And Jelovac, I. "GPs' Payment Contracts and Their Referral Practice.” Journal of Health Economics, Vol. 22 (2003), pp. 617-635.

O'Brien, D.P. AND SHAFFER, G. "Vertical Control with Bilateral Contracts.” RAND Journal of Economics, Vol. 23 (1992), pp. 299-308.

PARIKH, S. "How the Spider Catches the Fly: Referral Networks in the Plaintiffs' Personal Injury Bar." New York Law School Law Review, Vol. 51 (2006/2007), pp. 243-283.

PARK, I. "Cheap-Talk Referrals of Differentiated Experts in Repeated Relationships." RAND Journal of Economics, Vol. 36 (2005), pp. 391-411.

Pauly, M.V. "The Ethics and Economics of Kickbacks and Fee Splitting.” Bell Journal of Economics, Vol. 10 (1979), pp. 344-352.

Rebitzer, J.B. And Votruba, M.E. “Organizational Economics and Physician Practices.” NBER Working Paper no. 17535, 2011.

Reisinger, M. And TARAntino, E. "Vertical Integration, Foreclosure, and Productive Efficiency." RAND Journal of Economics, Vol. 46 (2015), pp. 461-479.

Rey, P. And Tirole, J. “A Primer on Foreclosure.” In M. Armstrong and R. Porter, eds., The Handbook of Industrial Organization. Amsterdam: North-Holland, 2007.

Schmidt, K.M. "The Costs and Benefits of Privatization: An Incomplete Contracts Approach." The Journal of Law, Economics \& Organization, Vol. 12 (1996), pp. 1-24.

Shumsky, R.A. And PInKeR, E.J. "Gatekeepers and Referrals in Services.” Management Science, Vol. 49 (2003), pp. 839-856.

Song, Z., Sequist, T.D., And BARnett, M.L. "Patient Referrals: A Linchpin for Increasing the Value of Care." JAMA, Vol. 312 (2014), pp. 597-598. 